\title{
SDSS-IV/SPIDERS: A catalogue of X-ray selected AGN properties
}

\section{Spectral properties and black hole mass estimates for SPIDERS SDSS DR14 type 1 AGN}

\author{
D. Coffey ${ }^{1}$, M. Salvato ${ }^{1}$, A. Merloni ${ }^{1}$, Th. Boller ${ }^{1}$, K. Nandra ${ }^{1}$, T. Dwelly ${ }^{1}$, J. Comparat $^{1}$, A. Schulze ${ }^{2}$, \\ A. Del Moro ${ }^{1}$, and D. P. Schneider ${ }^{3}$ \\ 1 Max-Planck-Institut für extraterrestrische Physik, Giessenbachstraße 1, 85748 Garching, Germany \\ e-mail: coffeydg@mpe.mpg.de \\ 2 National Astronomical Observatory of Japan, Mitaka, Tokyo 181-8588, Japan \\ 3 Department of Astronomy and Astrophysics, and the Institute for Gravitation and the Cosmos, The Pennsylvania State University, \\ 525 Davey Laboratory, University Park, PA 16802, USA
}

Received 31 July 2018 / Accepted 8 April 2019

\begin{abstract}
This work presents the catalogue of optical spectral properties for all X-ray selected SPIDERS active galactic nuclei (AGN) up to SDSS DR14. SPIDERS (SPectroscopic IDentification of eROSITA Sources) is an SDSS-IV programme that is currently conducting optical spectroscopy of the counterparts to the X-ray selected sources detected in the ROSAT all-sky survey and the XMM-Newton slew survey in the footprint of the Extended Baryon Oscillation Spectroscopic Survey (eBOSS). The SPIDERS DR14 sample is the largest sample of X-ray selected AGN with optical spectroscopic follow-up to date. The catalogue presented here is based on a clean sample of $73442 \mathrm{RXS}(\bar{z}=0.5)$ and 1157 XMM-Newton slew survey $(\bar{z}=0.4)$ type 1 AGN with spectroscopic coverage of the H $\beta$ and/or MgII emission lines. Visual inspection results for each object in this sample are available from a combination of literature sources and the SPIDERS group, which provide both reliable redshifts and source classifications. The spectral regions around the $\mathrm{H} \beta$ and MgII emission lines have been fit in order to measure both line and continuum properties, estimate bolometric luminosities, and provide black hole mass estimates using the single-epoch (or photoionisation) method. The use of both $\mathrm{H} \beta$ and MgII allows black hole masses to be estimated up to $z \simeq 2.5$. A comparison is made between the spectral properties and black hole mass estimates derived from $\mathrm{H} \beta$ and MgII using the subsample of objects which have coverage of both lines in their spectrum. These results have been made publicly available as an SDSS-IV DR14 value added catalogue.
\end{abstract}

Key words. quasars: emission lines - quasars: general - galaxies: active - catalogs - surveys

\section{Introduction}

A crucial requirement for understanding AGN evolution and demographics is the ability to select a sample for study in a complete and unbiased way. X-ray emission has been frequently used for AGN selection, and can distinguish the high energy emission associated with mass accretion by a black hole $(\mathrm{BH})$ from inactive galaxies and stars. Combining wide-area X-ray surveys with the ability to classify large numbers of objects spectroscopically via the Sloan Digital Sky Survey (SDSS; York et al. 2000; Gunn et al. 2006) provides a powerful tool for the study of AGN.

SPIDERS (SPectroscopic IDentification of eROSITA Sources; PIs Merloni and Nandra) is an SDSS-IV (Blanton et al. 2017) eBOSS (Dawson et al. 2016) subprogramme that is currently conducting optical spectroscopy of extragalactic X-ray detections in wide-area ROSAT and XMM-Newton surveys (Dwelly et al. 2017). Lying at the bright end of the X-ray source population, these sources will also be detected by eROSITA (Merloni et al. 2012; Predehl et al. 2016). The current SDSS DR14 (Abolfathi et al. 2018) SPIDERS sample is a powerful resource for the multiwavelength analysis of AGN. This work aims to capitalise on the wealth of information already available by providing detailed optical spectral measurements, as well as estimates of $\mathrm{BH}$ masses and Eddington ratios.
An accurate measurement of the central supermassive black hole $(\mathrm{SMBH})$ mass is necessary for the study of AGN and their coevolution with their host galaxies. BH mass has been found to scale with a number of host galaxy spheroid properties; stellar velocity dispersion (the $M_{\mathrm{BH}}-\sigma$ relation, e.g. Gebhardt et al. 2000; Merritt \& Ferrarese 2001; Tremaine et al. 2002), stellar mass (e.g. Magorrian et al. 1998), and luminosity (e.g. Kormendy \& Richstone 1995). These correlations suggest a symbiotic evolution of SMBHs and their host galaxies.

Reverberation mapping (RM) has been used to measure the approximate radius of the broad-line region (BLR) in AGN (e.g. Bahcall et al. 1972; Capriotti et al. 1982; Blandford \& McKee 1982; Peterson 1993; Bentz \& Katz 2015; Shen et al. 2015). This technique involves measuring the time delay between variations in the continuum emission, which is expected to arise from the accretion disk, and the induced variations in the broad emission lines. It was found that different emission lines have different time delays, which is expected if the BLR is stratified, with lines of lower ionisation being emitted further from the central ionising source (e.g. Gaskell \& Sparke 1986). For example, the high ionisation line CIV $\lambda 1549$ has a shorter time delay than $\mathrm{H} \beta$ (Peterson \& Wandel 2000).

The RM effort has also revealed a tight relationship between the continuum luminosity and the radius of the BLR 
(Kaspi et al. 2000; Bentz et al. 2006, 2009a). Therefore, by using the measured luminosity as a proxy for the BLR radius, and measuring the BLR line-of-sight velocity from the width of the broad emission lines, $\mathrm{BH}$ masses can be estimated from a single spectrum (Vestergaard 2002; McLure \& Jarvis 2002; Vestergaard \& Peterson 2006; Assef et al. 2011; Shen \& Liu 2012; Shen 2013). This approach is known as the single-epoch, or photoionisation, method.

Since $\mathrm{H} \beta$ is the most widely studied $\mathrm{RM}$ emission line it is therefore considered to be the most reliable line to use for single-epoch mass estimation. In addition, AGN $\mathrm{H} \beta$ emission lines typically exhibit a clear inflection point between the broad and narrow line components, making the virial full width at half maximum (FWHM) measurement relatively straightforward (see Sect. 3.3). The MgII line width correlates well with that of $\mathrm{H} \beta$ (see Sect. 7.2), and therefore MgII has also been used for single-epoch mass estimation (e.g. McLure \& Jarvis 2002). For SDSS spectra, either $\mathrm{H} \beta$ or $\mathrm{MgII}$ is visible in the redshift range $0 \leq z \lesssim 2.5$.

At higher redshifts, the broad, high-ionisation line CIV $\lambda 1549$ is available. The CIV line width does not correlate strongly with that of low ionisation lines (e.g. Baskin \& Laor 2005; Trakhtenbrot \& Netzer 2012) and this, along with the presence of a large blueshifted component (e.g. Richards et al 2002) makes it difficult to employ CIV for mass estimation. A number of calibrations have been developed which aim to improve the mass estimates derived from CIV (Denney 2012; Runnoe et al. 2013; Park et al. 2013; Coatman et al. 2017), however, whether CIV can provide reliable mass estimates when compared with low ionisation lines is still a subject of debate (see Mejía-Restrepo et al. 2018).

This paper is organised as follows: the selection of a reliable subsample of sources to be used for optical spectral fitting is discussed in Sect. 2. Section 3 describes the method used to fit the $\mathrm{H} \beta$ and $\mathrm{MgII}$ emission line regions. The methods for estimating $\mathrm{BH}$ mass and bolometric luminosity are discussed in Sect. 4. The X-ray flux measurements used in this work are discussed in Sect. 5. Section 6 describes where the catalogue containing the results of this work can be accessed. A comparison between the UV and optical spectral fitting results is given in Sect. 7. Section 8 provides a discussion of the sample properties, and finally, Sect. 9 includes a discussion of the reliability and limitations of the fitting procedure. In order to facilitate a direct comparison with previous studies based on X-ray surveys, a concordance flat $\Lambda$ CDM cosmology was adopted where $\Omega_{\mathrm{M}}=0.3$, $\Omega_{\Lambda}=1-\Omega_{\mathrm{M}}$, and $H_{0}=70 \mathrm{~km} \mathrm{~s}^{-1} \mathrm{Mpc}^{-1}$.

\section{Preparing the input catalogue}

\subsection{X-ray data}

The ROSAT sample used in this work is part of the second ROSAT all-sky survey (2RXS) catalogue (Boller et al. 2016), which has a limiting flux of $\sim 10^{-13} \mathrm{erg} \mathrm{cm}^{-2} \mathrm{~s}^{-1}$, which corresponds to a luminosity of $\sim 10^{43} \mathrm{erg} \mathrm{s}^{-1}$ at $z=0.5$. Compared to the first ROSAT data release (Voges et al. 1999), the $2 \mathrm{RXS}$ catalogue is the result of an improved detection algorithm, which uses a more detailed background determination relative to the original ROSAT pipeline. A full visual inspection of the 2RXS catalogue has been performed, which provides a reliable estimate of its spurious source content (see Boller et al. 2016). The first XMM-Newton slew survey catalogue release 1.6 (XMMSL1; Saxton et al. 2008) was also used in this work. This catalogue includes observations made by the European Photon
Imaging Camera (EPIC) pn detectors while slewing between targets, and has a limiting flux of $6 \times 10^{-13} \mathrm{erg} \mathrm{cm}^{-2} \mathrm{~s}^{-1}$ in the soft band, which corresponds to a luminosity of $5.8 \times 10^{44} \mathrm{erg} \mathrm{s}^{-1}$ at $z=0.5$.

\subsection{The SPIDERS programme}

The SPIDERS programme has been providing SDSS spectroscopic observations of $2 \mathrm{RXS}$ and XMMSL1 sources ${ }^{1}$ in the eBOSS footprint. Before the start of the eBOSS survey in 2014, the SPIDERS team compiled a sample of X-ray selected spectroscopic targets and submitted this sample for spectroscopic follow-up using the BOSS spectrograph as part of the eBOSS/SPIDERS subprogramme (see Dwelly et al. 2017, for further details on the SPIDERS programme). As of the end of eBOSS in February 2019, the eBOSS/SPIDERS survey has covered a sky area of $5321 \mathrm{deg}^{2}$. The SDSS DR14 SPIDERS sample presented in this work covers an area of $\sim 2200 \mathrm{deg}^{2}(\sim 40 \%$ of the final eBOSS/SPIDERS area).

The spectroscopic completeness achieved by the SPIDERS survey as of SDSS DR14 in the eBOSS area is $~ 53 \%$ for the sample as a whole, $\sim 63 \%$ considering only high-confidence X-ray detections (see Sect. 2.5 ), and $\sim 87 \%$ considering sources with high-confidence $\mathrm{X}$-ray detections and optical counterparts with magnitudes in the nominal survey limits $\left(17 \leq m_{\mathrm{Fiber} 2, i} \leq\right.$ 22.5). Outside the eBOSS area, the spectroscopic completeness of this sample is lower: $\sim 28 \%$ for the sample as a whole, $\sim 39 \%$ considering only high-confidence X-ray detections, and $\sim 57 \%$ considering sources with high-confidence X-ray detections and optical counterparts with magnitudes in the nominal survey limits. The spectroscopic completeness of the SDSS DR16 SPIDERS sample inside and outside the eBOSS area is expected to be similar to that of the sample presented here.

In addition to those targeted during eBOSS/SPIDERS, a large number of 2RXS and XMMSL1 sources received spectra during the SDSS-I/II (2000-2008; York et al. 2000) and the SDSSIII (Eisenstein et al. 2011) BOSS (2009-2014; Dawson et al. 2013) surveys. This paper includes spectra obtained by eBOSS/ SPIDERS up to DR14 (2014-2016) as well as spectra from SDSS-I/II/III.

\subsection{Identifying IR counterparts}

To identify SPIDERS spectroscopic targets, the Bayesian crossmatching algorithm "NWAY" (Salvato et al. 2018) was used to select AllWISE (Cutri et al. 2013) infrared (IR) counterparts for the 2RXS and XMMSL1 X-ray selected sources in the BOSS footprint. The AllWISE catalogue consists of data obtained during the two main survey phases of the Wide-field Infrared Survey Explorer mission (WISE; Wright et al. 2010) which conducted an all-sky survey in the $3.4,4.6,12$, and $22 \mu \mathrm{m}$ bands (magnitudes in these bands are denoted [W1], [W2], [W3], and [W4] respectively). The matching process used the colour-magnitude priors [W2] and [W2-W1] (see Dwelly et al. 2017) which, at the depth of the 2RXS and XMMSL1 surveys, can distinguish between the correct counterparts and chance associations. These colours would not be efficient if the 2RXS survey was much deeper (see Salvato et al. 2018, for a complete discussion). The

\footnotetext{
1 The SPIDERS programme targets both point-like and extended $\mathrm{X}$-ray sources. This work focuses on the counterparts to point-like X-ray detections, which are predominantly AGN, and therefore, the samples discussed in the subsequent paragraphs are derived from the SPIDERS-AGN programme.
} 


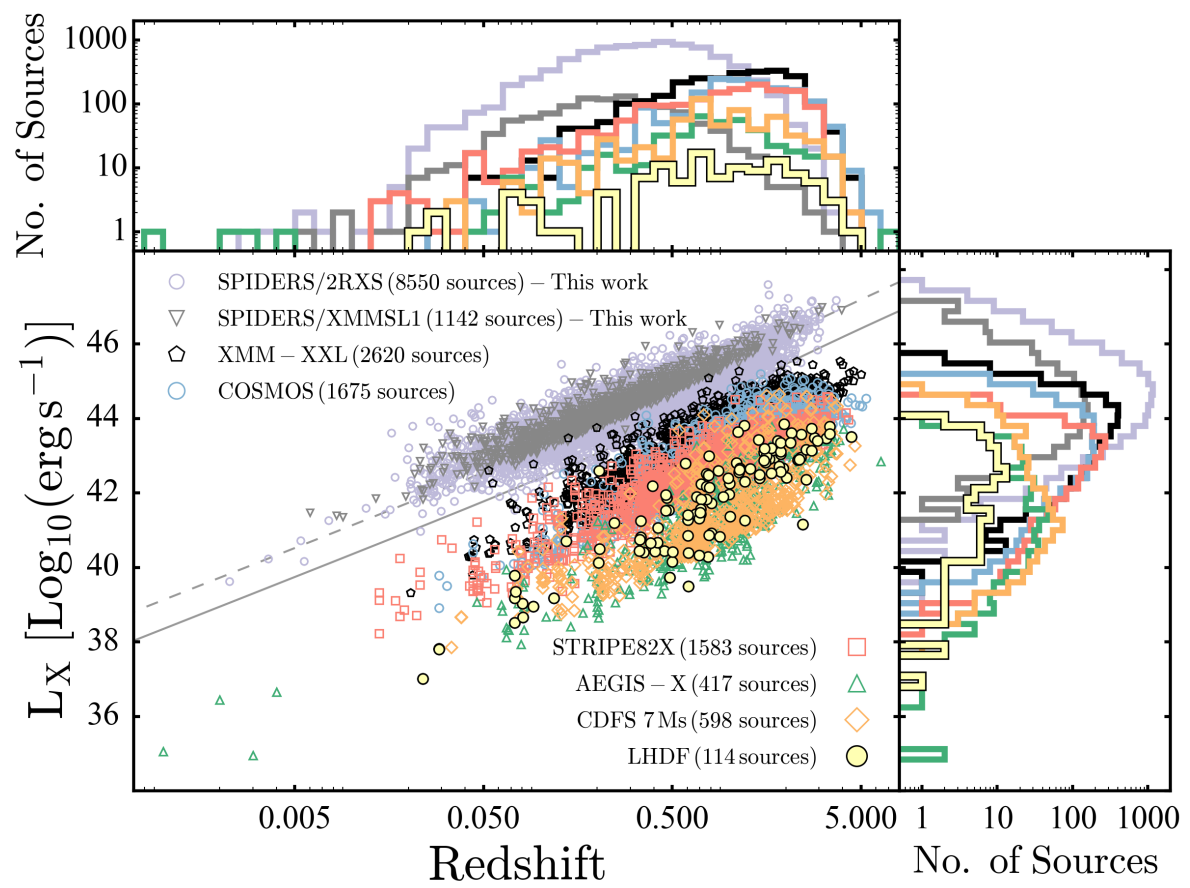

Fig. 1. Soft X-ray luminosity versus spectroscopic redshift for the samples presented in this work and the following previously published X-ray selected samples; XMM-XXL (Menzel et al. 2016; Liu et al. 2016), CDFS (Luo et al. 2017), STRIPE82X (LaMassa et al. 2016), COSMOS (Marchesi et al. 2016a,b; Civano et al. 2016), AEGIS-X (Nandra et al. 2015), and the Lockman Hole deep field (LHDF; Brunner et al. 2008; Fotopoulou et al. 2012). For each sample, the $0.5-2 \mathrm{keV}$ luminosities are shown, except for the $2 \mathrm{RXS}$ sample, where the $0.1-2.4 \mathrm{keV}$ luminosities are shown, and the XMMSL1 sample, where the 0.2-2 keV luminosities from Saxton et al. (2008) are shown. The detection limit for the 2RXS and XMMSL1 samples are shown by the solid and dashed grey lines respectively. The X-ray luminosities for the 2RXS sample are derived from the classical flux estimates described in Sect. 5, however it is noted here that some low count rate 2RXS sources do not have flux estimates. For sources that were detected in both 2RXS and XMMSL1, only the XMMSL1 luminosities are shown. Sources classified as stars have not been included in this figure.

resulting 2RXS and XMMSL1 catalogues with AllWISE counterparts contained 53455 and 4431 sources respectively. AllWISE positions were then matched to photometric counterparts, where available, in SDSS.

\subsection{Comparison with previous $X$-ray surveys}

Figure 1 displays the sources in the 2RXS and XMMSL1 samples which have spectroscopic redshifts and measurements of the soft X-ray flux. For comparison, a series of previously published $\mathrm{X}$-ray selected samples that have optical spectroscopic redshifts are also shown. The large number of sources present in the 2RXS and XMMSL1 samples motivated the optical spectroscopic analysis discussed in the following sections.

\subsection{Selecting a reliable subsample}

The selection of SPIDERS spectroscopic targets was discussed in detail by Dwelly et al. (2017). This section summarises the selection steps discussed in detail by Dwelly et al. (2017) and describes the additional cuts made in this work to select a sample for spectral analysis. The sequence of selection criteria used and the resulting sample size are shown in Fig. 2.

2RXS sources with an X-ray detection likelihood $($ EXI_ML) $\leq 10$ were excluded since these detections are considered highly uncertain with a spurious fraction $\geq 20 \%$ (see Boller et al. 2016). XMMSL1 sources with an X-ray detection likelihood (XMMSL_DET_ML_B0) $\leq 10$ were also excluded. Salvato et al. (2018; Fig. 1) show the distribution of flux with detection likelihood for both samples. These cuts returned 23245/53455 2RXS and 3803/4431 XMMSL1 sources.

The following cuts, which were described in detail in Dwelly et al. (2017), have also been applied to the sample:

- For each X-ray source, Salvato et al. (2018) give the probability, p_any, that a reliable counterpart exists among the possible AllWISE associations. Sources with $\mathrm{p} \_$any $<0.01$ were removed. This returned 23046/23245 2RXS and 3558/3803 XMMSL1 sources.

- For each X-ray source, the most probable AllWISE counterpart was chosen by selecting sources with match_flag $=1$. This returned 20585/23046 2RXS and 3321/3558 XMMSL1 sources.

- For each AllWISE counterpart, the brightest SDSS-DR13 photometric source within the AllWISE matching radius was selected using FLAG_SDSSv5b_best $=1$. This returned 19385/20585 2RXS and 3063/3321 XMMSL1 sources.

- Cases where the AllWISE-SDSS separation exceeded 3 arcsec were removed. This returned 18575/19385 2RXS and 2893/3063 XMMSL1 sources.

The results of these constraints are displayed in grey in Fig. 2. As shown above, sources with match_flag=1 were targeted; however, for $14 \%$ of the 2RXS sample and $10 \%$ of the XMMSL1 sample, more than one counterpart was highly likely. This implies that either the counterpart association was not reliable, or that the X-ray detection was the result of emission from multiple sources. These sources were not included in the discussion of optical spectral properties as a function of $\mathrm{X}$-ray properties in Sect. 8.3. After selecting the brightest SDSSDR13 (Albareti et al. 2017) photometric source, there were 

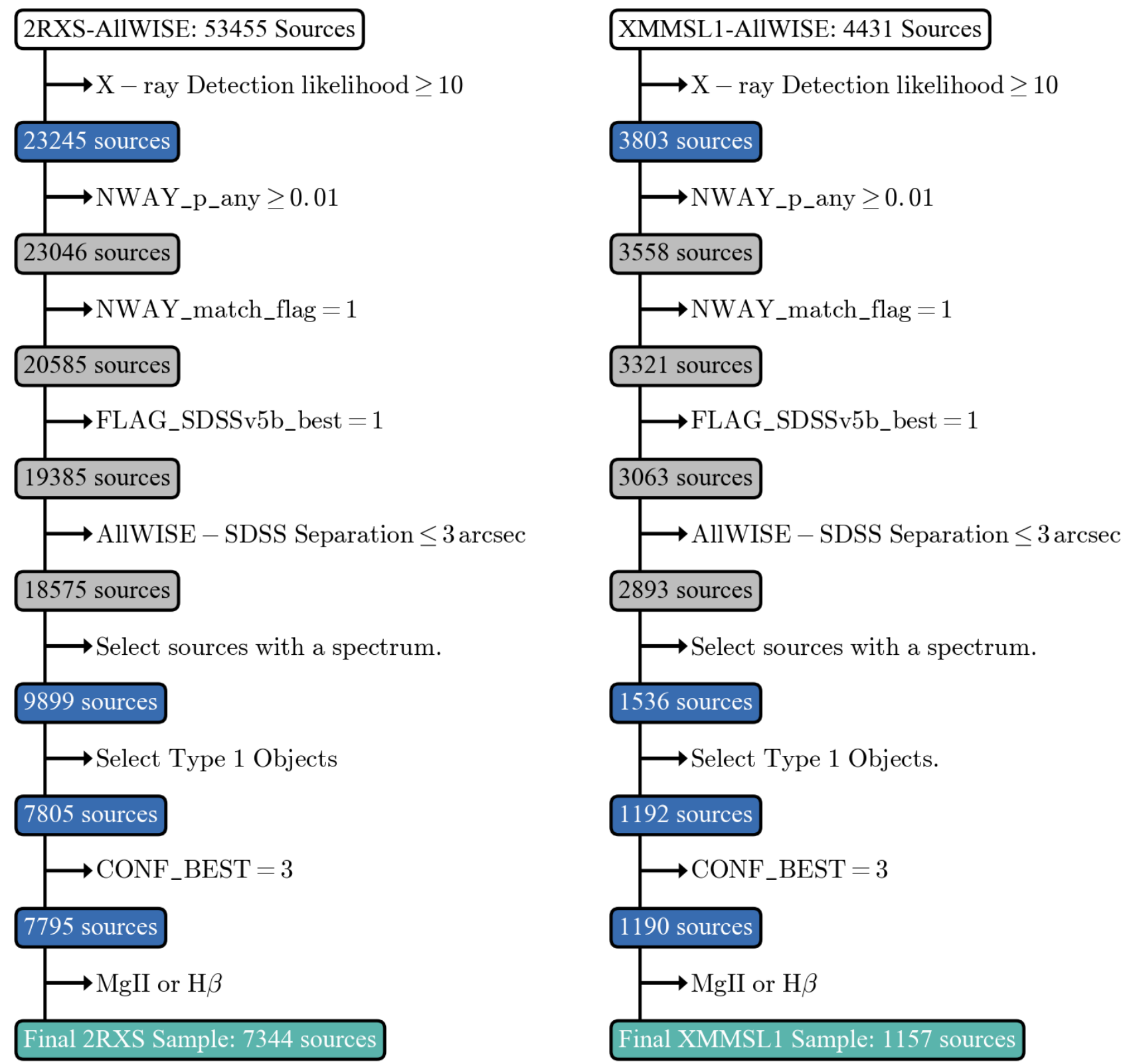

Fig. 2. Sequence of quality cuts applied to the $2 \mathrm{RXS}$ and XMMSL1 samples to produce the subsample used for spectral analysis. The starting points (2RXS-AllWISE and XMMSL1-AllWISE) are the full samples of 2RXS and XMMSL1 selected sources with AllWISE IR counterparts in the BOSS footprint (see Sect. 2.3). The steps in grey are those that have been discussed in Dwelly et al. (2017).

15 cases where two unique $2 \mathrm{RXS}$ sources were matched to the same AllWISE/SDSS counterpart and 3 cases where two unique XMMSL1 sources were matched to the same AllWISE/SDSS counterpart. These sources were also removed.

Of these samples with reliable SDSS photometric counterparts, 8777 2RXS and 1315 XMMSL1 sources have received spectra during SDSS-I/II/III while 1122 2RXS and 221 XMMSL1 sources have received spectra during the SPIDERS programme (including SEQUELS), resulting in a sample of 9899 2RXS and 1536 XMMSL1 sources with spectra as of DR14. The distribution of SDSS $i$ band fiber2 magnitudes for this sample (showing the different spectroscopic programmes) is presented in Fig. 3. Due to targeting constraints (as discussed in Sect. 2.2), the sample completeness is much lower outside of the nominal magnitude limits for the survey $\left(17 \leq m_{\mathrm{Fiber}, i} \leq 22.5\right.$ for eBOSS).

\subsection{Source classification}

Visual inspection results for each object in this sample are available from a combination of literature sources (Anderson et al. 2007; Plotkin et al. 2010; Schneider et al. 2010; Pâris et al. 2017) and the SPIDERS group. The SPIDERS visual inspection (see Dwelly et al. 2017, for further details) provides a visual confirmation of the SDSS pipeline redshift and object classification. The results of this inspection include a flag indicating the confidence of the redshift, "CONF_BEST", which can take the values 3 (highly secure), 2 (uncertain), 1 (poor/unusable), 0 (insufficient data). A confirmation of the source classification was also added during the visual inspection, which uses the categories QSO, broad absorption line QSO (BALQSO), blazar, galaxy, star, and none. Anderson et al. (2007) provide the broad line AGN (BLAGN) and narrow line AGN (NLAGN) 

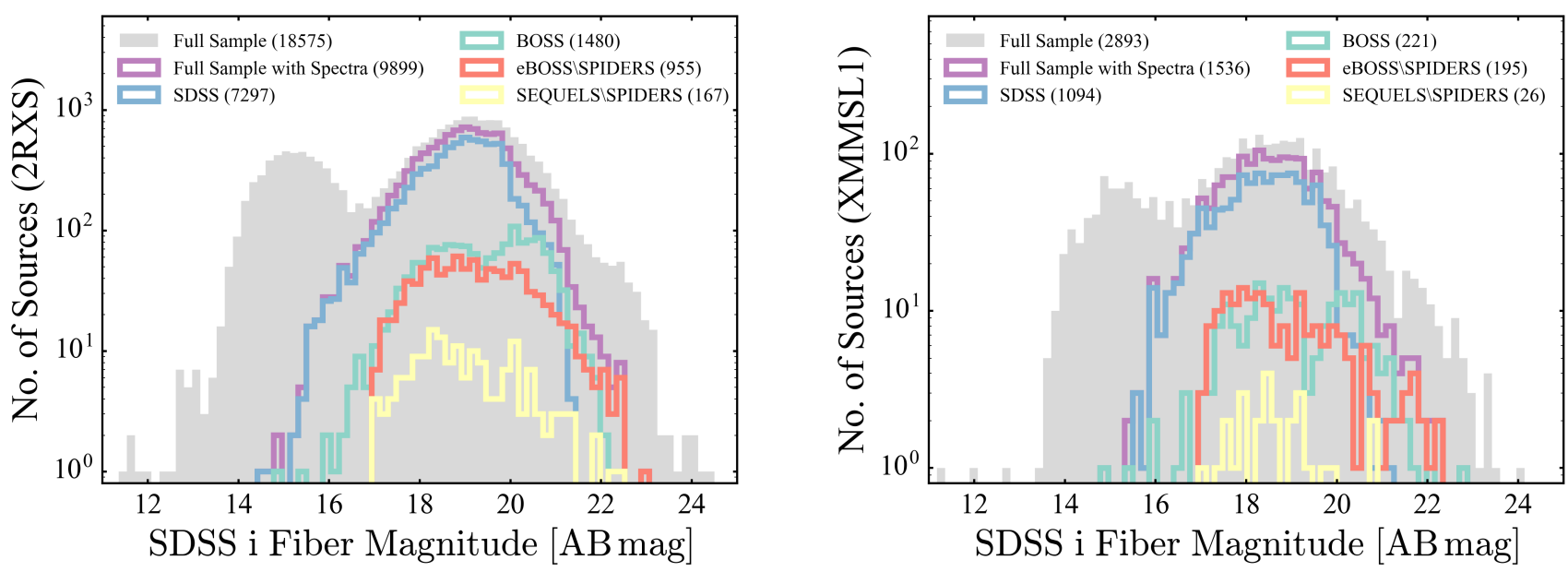

Fig. 3. Distribution of $i$-band fiber magnitudes (fiber2Mag). The coloured curves represent all of the sources with spectra, and the survey from which the spectra were taken. The grey histogram displays the X-ray sources with a reliable SDSS photometric counterpart, including stars which cannot be targets for spectroscopy due to their brightness.

classifications, which are defined based on the presence or absence of broad $\left(F W H M>1000 \mathrm{~km} \mathrm{~s}^{-1}\right)$ permitted emission lines.

The main goal of this work is to analyse the type 1 AGN in the SPIDERS sample, and therefore only sources that have been classified via their optical spectra as either "BLAGN" or "QSO" were selected for spectroscopic analysis. This returned 7805/9899 2RXS and 1192/1536 XMMSL1 sources. Since the categories "BLAGN" and "QSO" are based on different classification criteria, there will be some overlap between the two sets of sources. Therefore, no distinction will be made between the two categories; instead, both sets of objects will be considered type $1 \mathrm{AGN}$ in this work.

\subsection{Contamination from starburst galaxies}

Although our sample probes luminosity ranges typically associated with AGN emission, starburst galaxies are also powerful X-ray sources and may be present as contaminants in our AGN sample. The X-ray emission from starburst galaxies is expected to originate from a number of energetic phenomena including supernova explosions and X-ray binaries (e.g. Persic \& Rephaeli 2002). Therefore, the X-ray emission from starburst galaxies can be expected to be correlated with the star formation rate (SFR). Using their sample of luminous infrared galaxies, and a sample of nearby galaxies from Ranalli et al. (2003), Pereira-Santaella et al. (2011) found that the total SFR is related to the soft X-ray luminosity as follows:

$S F R_{\mathrm{IR}+\mathrm{UV}}\left(M_{\odot} \mathrm{yr}^{-1}\right)=3.4 \times 10^{-40} L_{0.5-2 \mathrm{keV}}\left(\mathrm{erg} \mathrm{s}^{-1}\right)$.

Ilbert et al. (2015), Fig. 3, show the specific SFR for the COSMOS (Scoville et al. 2007) and GOODS (Giavalisco et al. 2004) surveys for a series of redshift bins in the range $0.2<z<$ 1.4. The peak of the redshift distribution of the 2RXS/XMMSL1 samples presented in this work is $\sim 0.25$. Therefore, assuming that the COSMOS/GOODS sample in the redshift bin $0.2-0.4$ is a good representative of the 2RXS/XMMSL1 samples, the upper limit on the SFR that can be expected for galaxies in our sample is $\sim 50 M_{\odot} \mathrm{yr}^{-1}$. According to Eq. (1), this corresponds to a soft X-ray luminosity of $\sim 10^{41} \mathrm{erg} \mathrm{s}^{-1}$, which is below the lower range probed by our samples $\left(\sim 10^{42} \mathrm{erg} \mathrm{s}^{-1}\right.$, see Fig. 1).

\subsection{Redshift constraints}

Using the "CONF_BEST" flag, sources with uncertain redshift or spectral classification (identified during the visual inspection of the sample) were also removed. This process resulted in a sample of 7795/7805 2RXS sources and 1190/1192 XMMSL1 sources. In the spectral fitting procedure (described in Sect. 3), the $\mathrm{H} \beta$ and $\mathrm{MgII}$ lines were fit independently. Sources with $\mathrm{H} \beta$ and MgII present in their optical spectrum were selected using the following logic:

$$
\begin{array}{r}
\mathrm{H}_{\beta}:\left(\mathrm{SN} \_ \text {MEDIAN_ALL }>5\right) \& \& \\
\left(\left((\mathrm{INSTRUMENT}==\mathrm{SDSS}) \& \&\left(0<\mathrm{Z} \_ \text {BEST }<0.81\right)\right) \|\right. \\
\left.\left((\mathrm{INSTRUMENT}==\mathrm{BOSS}) \& \&\left(0<\mathrm{Z} \_ \text {BEST }<1.05\right)\right)\right)
\end{array}
$$

$$
\begin{array}{r}
\text { MgII }:(\text { SN_MEDIAN_ALL }>5) \& \& \\
(((\text { INSTRUMENT }==\text { SDSS }) \& \&(0.45<\text { Z_BEST }<2.1)) \| \\
((\text { INSTRUMENT }==\text { BOSS }) \& \&(0.38<\text { Z_BEST }<2.5)))
\end{array}
$$

Different redshift ranges have been used because the BOSS spectrograph has a larger wavelength coverage than the SDSS spectrograph. In some cases, parts of the fitting region will have been redshifted out of the SDSS/BOSS spectrograph range (Smee et al. 2013), and therefore will not be fit. However, the redshift limits where chosen so that both samples contain the broad lines used for estimating BH mass. Sources with a median signal-to-noise ratio $(\mathrm{S} / \mathrm{N})$ less than or equal to five per resolution element were excluded from the spectral analysis since for these sources the broad line decomposition and resulting $\mathrm{BH}$ mass estimates may be unreliable (see Denney et al. 2009; Shen et al. 2011).

Table 1 lists the numbers of sources with spectral coverage of either $\mathrm{H} \beta$ or MgII, while Fig. 4 shows the redshift distribution of these sources. There are 711 cases where the same optical counterpart was detected by both 2RXS and XMMSL1. The final combined (2RXS and XMMSL1) sample for spectral analysis contains 7790 unique type 1 sources.

\section{Spectral analysis}

A series of scripts have been written to perform spectral fits using the MPFIT least-squares curve fitting routine (Markwardt 2009). Each spectrum was corrected for Milky Way extinction using the 
Table 1. The coverage of the $\mathrm{H} \beta$ and $\mathrm{MgII}$ emission lines in the two samples used in this work.

\begin{tabular}{lcccc}
\hline \hline & $\mathrm{MgII}$ & $\mathrm{H} \beta$ & $\mathrm{H} \beta$ and MgII & $\mathrm{H} \beta$ or MgII \\
\hline 2RXS & 3310 & 6268 & 2234 & 7344 \\
XMMSL1 & 314 & 1070 & 227 & 1157 \\
Total & 3473 & 6654 & 2337 & 7790 \\
\hline
\end{tabular}

Notes. There are 711 sources which were detected in both the 2RXS and XMMSL1 surveys. The "total" row lists the total number of unique sources obtained from combining the 2RXS and XMMSL1 samples.

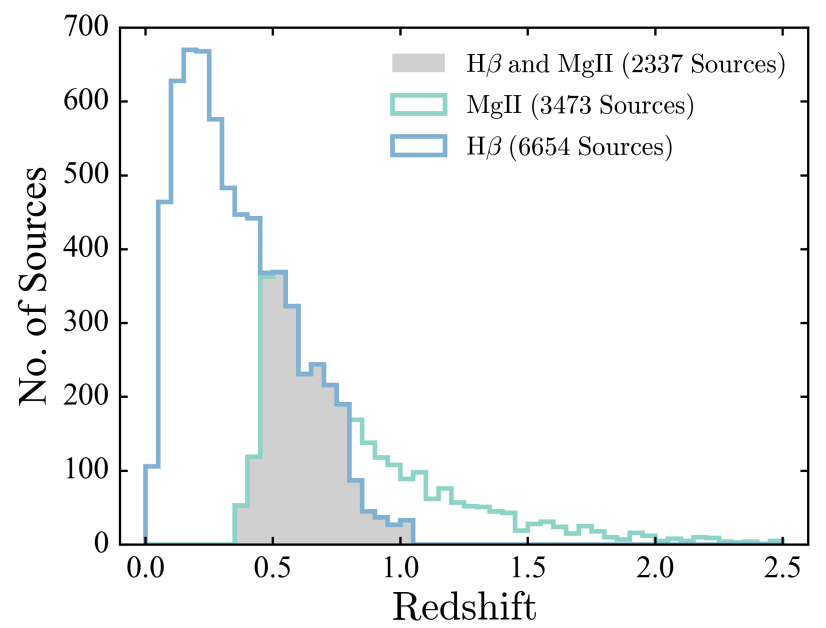

Fig. 4. Redshift distribution of the sample of type 1 AGN with coverage of $\mathrm{H} \beta$ and/or MgII.

extinction curve from Cardelli et al. (1989), and the dust map from Schlegel et al. (1998), with an $R_{\mathrm{V}}=3.1$. No attempt has been made to estimate and correct for the intrinsic (host) extinction of each source ${ }^{2}$. Measured line widths were corrected for the resolution of the SDSS/BOSS spectrographs. The $\mathrm{H} \beta$ and MgII emission line regions were fit independently using similar methods described in the following sections ${ }^{3}$.

\subsection{Iron emission template}

AGN typically exhibit FeII emission consisting of a large number of individual lines across the optical and UV regions of the spectrum. These lines appear to be blended, probably due to the motion of the gas from which they are emitted, and the magnitude of this broadening varies significantly from source to source. The presence of FeII emission in the optical and UV portions of the spectrum can be a significant complication when attempting to accurately measure line profiles. Therefore it is crucial that the model used to derive line widths for $\mathrm{BH}$ mass measurements also accounts for the nearby FeII emission.

Figure 5 shows the two FeII templates used in this work; the Vestergaard \& Wilkes (2001) and Boroson \& Green (1992) templates used for the UV and optical regions of the spectrum, respectively. Both of these templates have been derived from the narrow line Seyfert 1 galaxy I Zwicky 1 which, due to its bright

2 Also note that extinction laws (e.g. Calzetti and Prevot) are based on samples of nearby SB and irregular type galaxies. Due to the lack of nearby passive galaxies, an extinction law for these galaxy types is not yet available.

3 For each model parameter, the 1-sigma uncertainties from MPFIT were adopted.
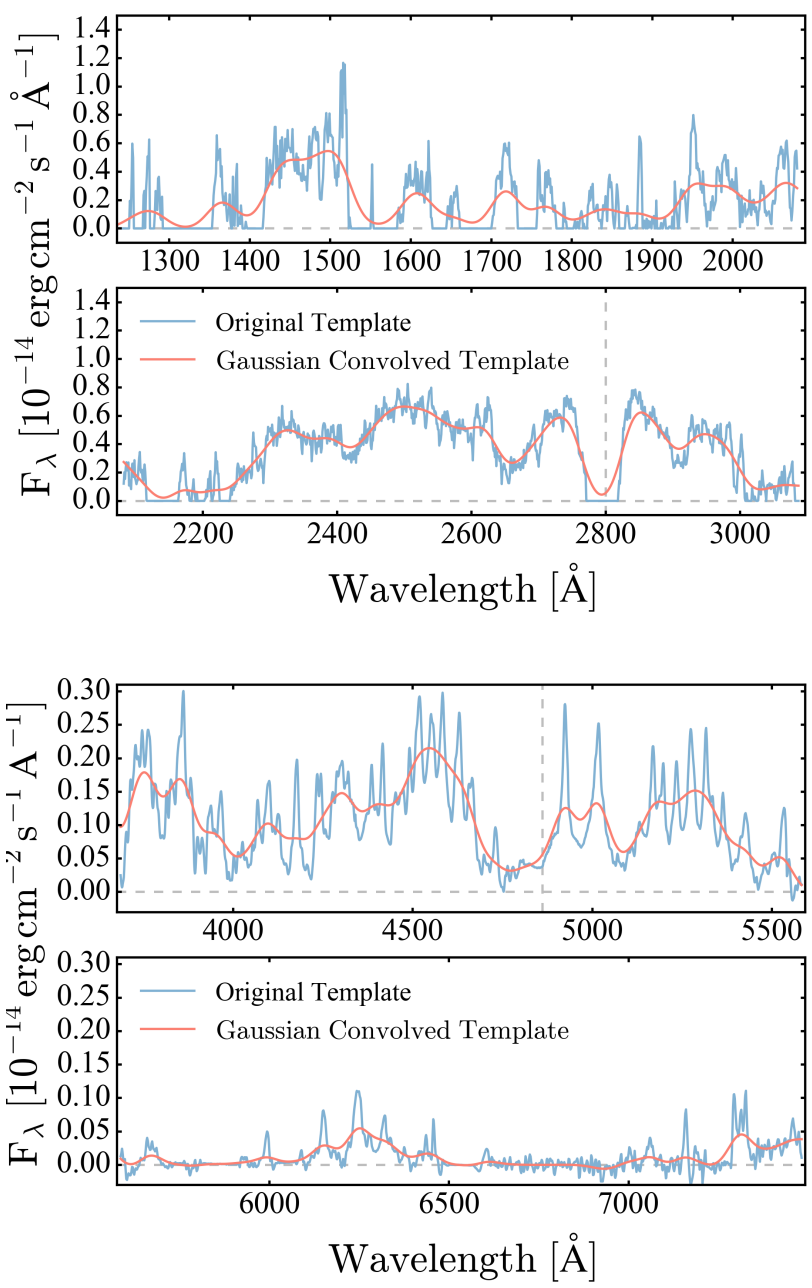

Fig. 5. Upper panel: Vestergaard \& Wilkes (2001) FeII template which was used when fitting the MgII emission line region. Lower panel: Boroson \& Green (1992) FeII template which was used when fitting the $\mathrm{H} \beta$ emission line region. In both cases, the original template is shown (blue) along with the same template convolved with a Gaussian with $F W H M=4000 \mathrm{~km} \mathrm{~s}^{-1}$ (red). The vertical dashed lines correspond to the position of $\mathrm{MgII}$ and $\mathrm{H} \beta$ at $2800 \AA$ and $4861 \AA$.

FeII emission and narrow emission lines, is an ideal candidate for generating the FeII template. In order to model the observed blending of the FeII emission, the templates were convolved with a Gaussian whose width was included as a free parameter in the fitting procedure.

\section{2. $H \beta$}

The region from $4420-5500 \AA$ was fit for each spectrum. The continuum model consisted of a power law, a galaxy template, and the Boroson \& Green (1992) FeII emission template. The FeII template was convolved with a Gaussian while fitting, and the width of this Gaussian, along with the normalisation of the template were included as free parameters in the fit (see Sect. 3.1). Previous spectral analyses of AGN spectra have assumed an early-type galaxy component in the model (Calderone et al. 2017). Following this method, we use an earlytype SDSS galaxy template ${ }^{4}$ in the fit, and the normalisation of

4 Template number 24 on http://classic.sdss.org/dr5/ algorithms/spectemplates/. 

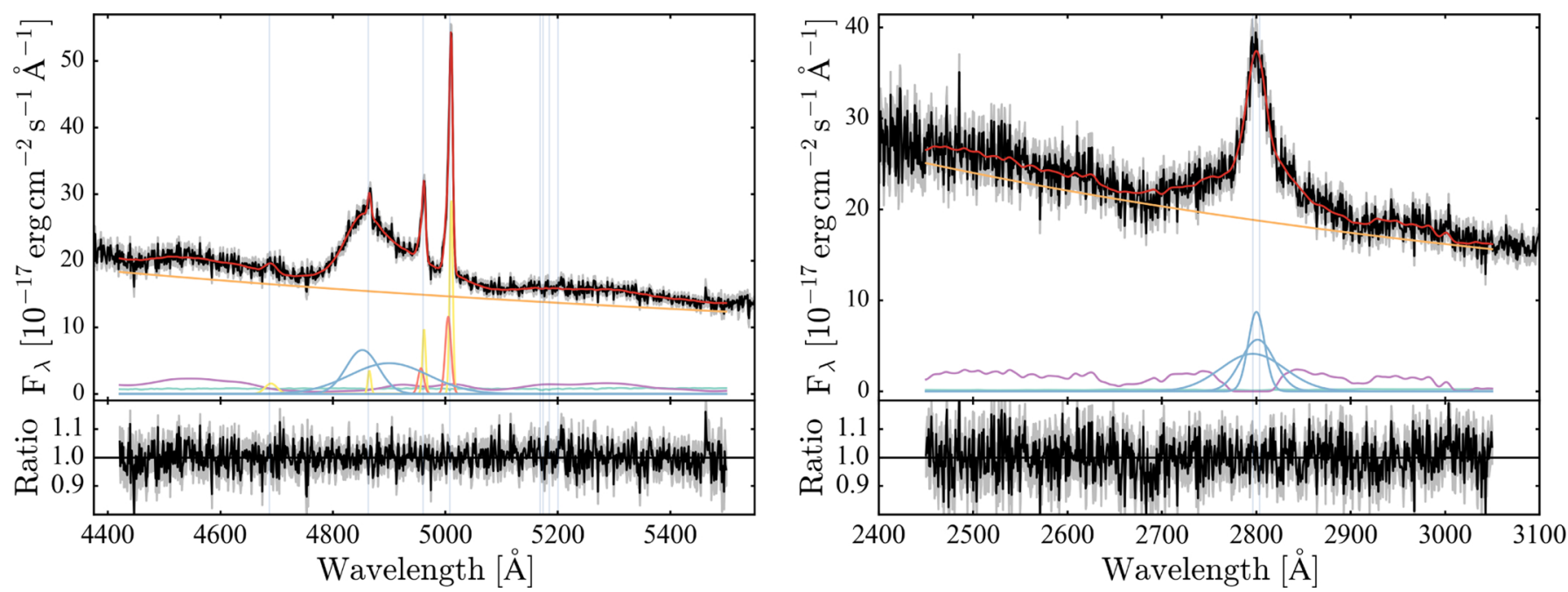

Fig. 6. Examples of model fits to the $\mathrm{H} \beta$ (left panel; plate $=1159$, MJD $=52669$, fiber $=470)$ and $\mathrm{MgII}$ (right panel; plate $=423$, MJD $=51821$, fiber $=250)$ spectral regions. The model components are colour-coded as follows; power law (orange), iron emission (violet), broad lines (blue), narrow lines (yellow), [OIII] shifted wings (red), and the total model (red). The panels beneath the spectra show the data/model ratio.

this template as well as the normalisation and slope of the power law were also free parameters. The use of a single, early-type galaxy template is an approximation, however, it is considered to be justified since AGN are typically found to reside in bulge dominated galaxies (e.g. Grogin et al. 2005; Pierce et al. 2007), and the spectroscopic fiber collects emission mostly from the bulge (which is characterised by an old stellar population) and the active nucleus.

The [OIII] $\lambda 4959$ and [OIII] $] \lambda 5007$ narrow lines were each fit with two Gaussians, one used to fit the narrow core, and an additional Gaussian to account for the presence of blue-shifted wings which are often detected (Boroson 2005). A single Gaussian was used to fit the HeII $\lambda 4686$ emission line. To avoid overfitting the $\mathrm{H} \beta$ line, the fitting process was run four times, with one, two, three, and four ${ }^{5}$ Gaussian components used to fit the $\mathrm{H} \beta$ line. For each fit, the velocity width and peak wavelength of one of the Gaussian components was fixed to that of [OIII] $\lambda 4959$ and [OIII] $\lambda 5007$ in order to aid the identification of the narrow $\mathrm{H} \beta$ component. The normalisation ratio of the [OIII] $\lambda 4959$ and [OIII] $\lambda 5007$ lines was fixed to the expected value of 1:3 (e.g. Storey \& Zeippen 2000). The best-fit model was then selected using the Bayesian information criterion (BIC; Schwarz 1978), which can be written as

$\mathrm{BIC}=\ln (n) k+\chi^{2}$

where $n$ is the number of data points, $k$ is the number of model parameters, and $\chi^{2}$ is the chi-square of the fit. The preferred model is that with the lowest BIC. An example of a fit to the $\mathrm{H} \beta$ spectral region is shown in the left panel of Fig. 6 .

\subsection{Broad line decomposition}

The narrow $\mathrm{H} \beta$ and [OIII] components are required to have widths $\leq 800 \mathrm{~km} \mathrm{~s}^{-1}$. Any of the additional Gaussians used to fit $\mathrm{MgII}$ and $\mathrm{H} \beta$ with $F W H M>800 \mathrm{~km} \mathrm{~s}^{-1}$ are considered

\footnotetext{
5 Three broad Gaussians are used in addition to a single narrow component to account for the three distinct broad components that are expected to be present (see Sect. 8.1) in at least some sources (Marziani et al. 2010).
}

"broad". This threshold of $800 \mathrm{~km} \mathrm{~s}^{-1}$ is taken from the approximate division between broad and narrow FWHM distributions in the lower panels of Fig. 12. The virial FWHM used for BH mass estimation is the FWHM of the line profile defined by the sum of these broad Gaussian components (see Fig. 7). A major challenge with using the single-epoch method for estimating $\mathrm{BH}$ mass is decomposing the broad and narrow components of the line in order to measure the virial FWHM. Figure 7 presents an example of the decomposition of a broad $\mathrm{H} \beta$ line. In this case, the narrow $\mathrm{H} \beta$ core can be easily distinguished and removed before measuring the virial FWHM. However, there are many cases where the broad and narrow components are blended, making it difficult to successfully identify the appropriate virial FWHM. There are also cases where there is a clear distinction between two broad line components that are shifted in wavelength relative to each other (known as "double-peaked emitters"). How one should interpret the single-epoch BH mass estimates for these unusual objects is uncertain (also see Sect. 9.2).

\subsection{Mgll}

The region from $2450-3050 \AA$ was fit for each spectrum. As in the case of the $\mathrm{H} \beta$ fits, a power law, an early-type galaxy template (5 Gyr old elliptical galaxy; Silva et al. 1998; Polletta et al. 2007), and the Vestergaard \& Wilkes (2001) FeII emission template were used to fit the continuum. Again, the FeII template normalisation, and width of the Gaussian smoothing applied to the template, were included as free parameters in the fit. The MgII line is a doublet; however, due to the close spacing and virial broadening of the lines, it usually appears as a single broad component in AGN spectra. The narrow MgII line cores are usually not observed in AGN spectra, therefore the MgII profile was fit using three broad Gaussians. An example of a fit to the MgII spectral region is presented in the right panel of Fig. 6.

\section{Bolometric luminosity and $\mathrm{BH}$ mass estimation}

Bolometric luminosities were estimated from the monochromatic luminosities using the bolometric corrections from 


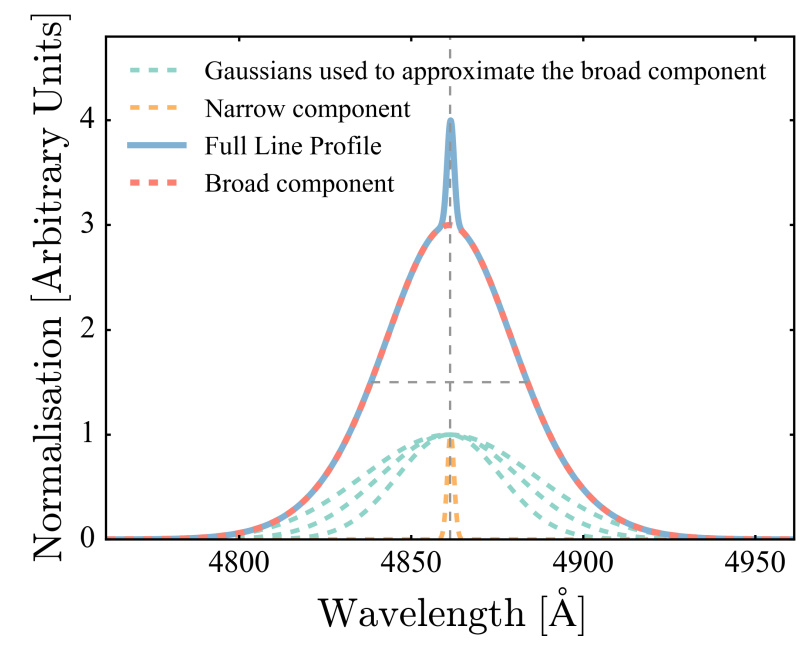

Fig. 7. An example of the decomposition of a typical AGN $\mathrm{H} \beta$ line (plate $=7276, M J D=57061$, fiber $=470)$. The horizontal dashed line represents the FWHM used for BH mass estimation. The vertical dashed line indicates the rest-frame wavelength of $\mathrm{H} \beta$. See Sect. 3.3 for further details.

Richards et al. (2006), Shen et al. (2011):

$L_{\mathrm{Bol}}=5.15 L_{3000 \AA}$

$L_{\mathrm{Bol}}=9.26 L_{5100 \AA}$.

These bolometric corrections have been derived using mean AGN SEDs; however, Richards et al. (2006) note that using a bolometric correction resulting from a single mean SED can result in bolometric luminosities with inaccuracies up to $50 \%$.

Under the assumption that the BLR gas is virialised, the single-epoch method can be used to estimate BH mass as follows:

$\log \left(\frac{M_{\mathrm{BH}}}{M_{\odot}}\right)=\mathrm{A}+\mathrm{B} \log \left(\frac{\lambda L_{\lambda}}{10^{44} \mathrm{erg} \mathrm{s}^{-1}}\right)+\mathrm{C} \log \left(\frac{F W H M}{\mathrm{~km} \mathrm{~s}^{-1}}\right)$

where $L_{\lambda}$ is the monochromatic luminosity at wavelength $\lambda$, and FWHM is the full width at half maximum of the broad component of the emission line. A, B, and C are constants that are calibrated using RM results and vary depending on which line is used.

Over the years, many groups have provided calibrations of Eq. (2) for MgII and $\mathrm{H} \beta$. In this work, the calibrations from Vestergaard \& Peterson (2006) and Assef et al. (2011) are used for $\mathrm{H} \beta$. Vestergaard \& Peterson (2006) based their work on an updated study of the $R_{\mathrm{BLR}}-L$ relationship (Kaspi et al. 2005; Bentz et al. 2006) and a reanalysis of the RM mass estimates (Peterson et al. 2004) and therefore presented an improved mass calibration relative to previous studies. Assef et al. (2011) provide a mass calibration that is based on the $R_{\mathrm{BLR}}-L$ relationship from Bentz et al. (2009b). Vestergaard \& Peterson (2006) and Assef et al. (2011) both provide similar calibrations for singleepoch $\mathrm{H} \beta$ mass estimation, as can be seen from the left panel of Fig. 8.

The Shen \& Liu (2012) calibration is used in this work for MgII. This calibration is based on a sample of 60 highluminosity $\left(L_{5100 \AA}>10^{45.4} \mathrm{erg} \mathrm{s}^{-1}\right)$ quasars in the redshift range 1.5-2.2. Shen \& Liu (2012) use the Vestergaard \& Peterson (2006) mass estimates as a reference when determining their MgII calibration. The centre and right panels of Fig. 8 show the comparison between the Shen \& Liu (2012) MgII calibration and the $\mathrm{H} \beta$ calibrations from Vestergaard \& Peterson (2006) and Assef et al. (2011). These calibrations agree reasonably well, with the standard deviation $\sigma \simeq 0.3$ in both cases, which is likely due to the fact that these $\mathrm{BH}$ mass estimates were derived using two different emission lines. A list of the three BH mass calibrations used in this work is given in Table 2.

BH masses were computed for each of these calibrations and are included in the catalogue (see Appendix A). BH masses were only estimated for sources with a detected broad line component (see Sect. 3.3). These BH mass estimates were then used to estimate the Eddington luminosity and the Eddington ratio

$$
\begin{aligned}
L_{\mathrm{Edd}} & =4 \pi c G M_{\mathrm{BH}} m_{\mathrm{p}} / \sigma_{\mathrm{T}} \\
\lambda_{\mathrm{E}} & =L_{\mathrm{Bol}} / L_{\mathrm{Edd}}
\end{aligned}
$$

where $c$ is the speed of light, $G$ is the gravitational constant, $M_{\mathrm{BH}}$ is the $\mathrm{BH}$ mass, $m_{\mathrm{p}}$ is the proton mass, and $\sigma_{\mathrm{T}}$ is the Thomson scattering cross-section.

\section{X-ray flux estimates}

Since X-ray detections are available for all objects in this sample, $\mathrm{X}$-ray flux estimates have also been included in the catalogue. XMMSL1 fluxes in the $0.2-12 \mathrm{keV}$ range from Saxton et al. (2008) are included. Saxton et al. (2008) convert the XMMSL1 count rates to fluxes using a spectral model consisting of an absorbed power law with a photon index of 1.7 and $N_{\mathrm{H}}=$ $3 \times 10^{20} \mathrm{~cm}^{-2}$.

Many of the sources in the 2RXS sample have flux measurements close to the ROSAT flux limit $\left(\sim 10^{-13} \mathrm{erg} \mathrm{cm}^{-2} \mathrm{~s}^{-1}\right)$. Therefore, when estimating fluxes for this sample, it was necessary to correct for the Eddington bias. This was done by adopting a Bayesian method to derive a probability distribution of fluxes based on the known distribution of AGN as a function of flux. Following Kraft et al. (1991), Laird et al. (2009), and Georgakakis \& Nandra (2011), the probability of a source having flux $f_{\mathrm{X}}$, given an observed number of counts $C$, is

$$
P\left(f_{\mathrm{X}}, C\right)=\frac{T^{C} e^{-T}}{C !} \pi\left(f_{\mathrm{X}}\right)
$$

where $C$ is the total number of observed source and background counts, $T$ is the mean expected total counts in the detection cell for a given flux, and $\pi\left(f_{\mathrm{X}}\right)$ is the prior, which is the distribution of AGN per unit X-ray flux interval. The exact expression for the prior was taken from Georgakakis et al. (2008), Eq. (1).

Source and background counts were taken from the 2RXS catalogue (Boller et al. 2016). A flux-count rate conversion factor, which was required to estimate $T$ in Eq. (3), was derived using XSPEC (Arnaud 1996) assuming a model consisting of a power law (with $\Gamma=2.4$ following Dwelly et al. 2017) absorbed by the Milky Way column density. This method was used to estimate the flux in the full ROSAT band $(0.1-2.4 \mathrm{keV})$ as well the monochromatic flux at $2 \mathrm{keV}$.

The fluxes resulting from the method described above with and without applying the prior (termed "Bayesian" and "classical", respectively) are compared in Fig. 9. The disagreement between the two flux estimates increases with decreasing flux, which is expected since, without the prior, the classical method fails to account for the Eddington bias. Low count rate sources in this sample would be assigned unrealistically low Bayesian fluxes. To avoid this, the flux was left as undetermined when the Bayesian flux estimate was more than a factor of ten smaller than the classical flux estimate. 

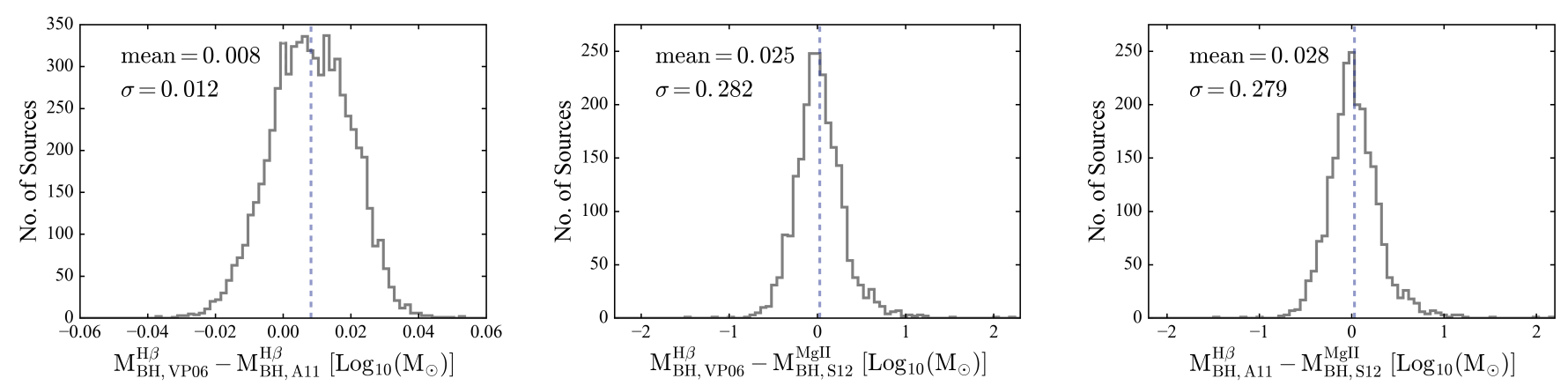

Fig. 8. Differences between the BH mass calibrations used in this work (see Sect. 4 for further details). The vertical blue lines indicate the mean value of each distribution. The standard deviation, $\sigma$, of each distribution is also shown.

Table 2. BH mass calibrations used in this work.

\begin{tabular}{lcccc}
\hline \hline & $\mathrm{A}$ & $\mathrm{B}$ & $\mathrm{C}$ & Reference \\
\hline $\mathrm{MgII}, L_{3000 \AA}$ & 1.816 & 0.584 & 1.712 & Shen \& Liu (2012) \\
$\mathrm{H}_{\beta}, L_{5100 \AA}$ & 0.91 & 0.5 & 2 & Vestergaard \& Peterson (2006) \\
$\mathrm{H}_{\beta}, L_{5100 \AA}$ & 0.895 & 0.52 & 2 & Assef et al. (2011) \\
\hline
\end{tabular}

Notes. A, B, and C are the calibration constants for single-epoch mass estimation (see Eq. 2).

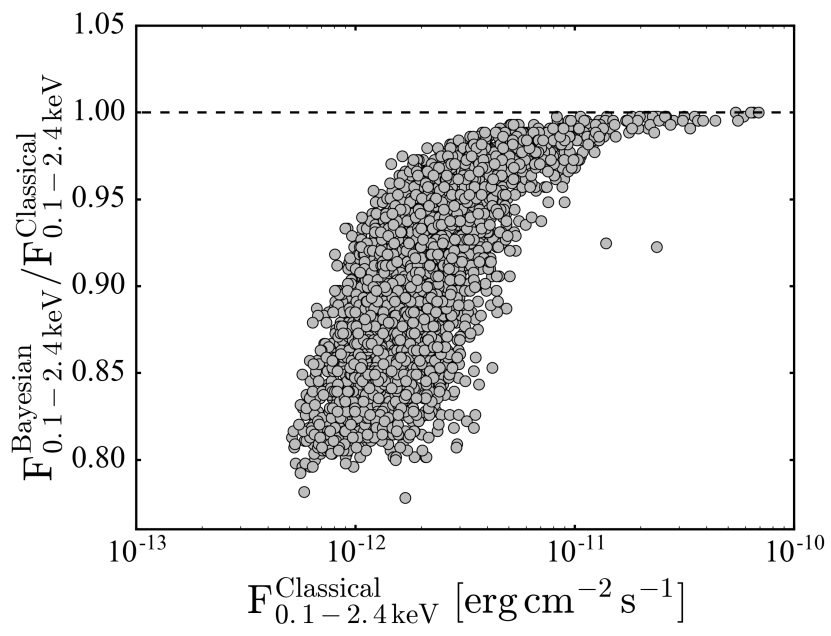

Fig. 9. Comparison between the classical and Bayesian methods for estimating 2 RXS fluxes. The deviation from a ratio of one at fainter fluxes results from the attempt to correct for the effect of the Eddington bias (see Sect. 5 for further details).

\section{Accessing the data}

The results from the spectral analysis discussed above, along with X-ray flux measurements and visual inspection results, have been made available in an SDSS DR14 value added catalogue $^{6}$. Additionally, an extended version of the catalogue will be maintained at http://www.mpe.mpg.de/ XraySurveys/SPIDERS/SPIDERS_AGN/. The column description for the catalogue is given in Appendix A.

\section{Comparing the UV and optical fitting results}

A subsample of sources have spectral measurements available from both the $\mathrm{MgII}$ and $\mathrm{H} \beta$ spectral regions. In order to test the

\footnotetext{
6 Available at http://www.sdss.org/dr14/data_access/ value-added-catalogs/
}

consistency of the independent fits to these two regions, properties measured from both were compared.

\section{1. $L_{2500 \AA}-L_{5100 \AA}$ relation}

A subsample of AGN whose spectra cover the rest-frame wavelengths $2500 \AA$ and $5100 \AA$ was selected using the following criteria:

$(($ INSTRUMENT $=$ SDSS $) \& \&(0.52<$ redshift $<0.8)) \|$ $(($ INSTRUMENT $=$ BOSS $) \& \&(0.46<$ redshift $<1.04))$.

Of this sample, 1718 sources had reliable measurements of both $L_{2500 \AA}$ and $L_{5100 \AA}$. The $L_{2500 \AA}-L_{5100 \AA}$ relation was fit using the LINMIX (Kelly 2007) package. LINMIX is a Bayesian linear regression algorithm that accounts for uncertainties in both dependent and independent variables, as well as non-detections. The upper left panel of Fig. 10 shows the $L_{2500 \AA}-L_{5100 \AA}$ distribution and the best-fit relation

$\log _{10}\left(L_{5100 \AA}\right)=(0.841 \pm 0.007) \log _{10}\left(L_{2500 \AA}\right)-(6.0 \pm 0.3)$

with a regression intrinsic scatter of 0.0151 . The comparison between the estimated bolometric luminosities derived from the $3000 \AA$ and $5100 \AA$ monochromatic fluxes is shown in the upper right panel of Fig. 10. Equation (4) can be used to estimate $L_{2500 \AA}$ from $L_{5100 \AA}$, which allows low redshift sources to be included in the $\alpha_{\mathrm{OX}}$ analysis discussed in Sect. 8.3.

\subsection{Comparing Mgll and $H \beta$ FWHM measurements}

A subsample of AGN whose spectra cover the broad $\mathrm{H} \beta$ and $\mathrm{MgII}$ emission lines was selected using the following criteria

$$
\begin{gathered}
((\text { INSTRUMENT }=\text { SDSS }) \& \&(0.45<\text { redshift }<0.81)) \| \\
((\text { INSTRUMENT }=\text { BOSS }) \& \&(0.38<\text { redshift }<1.05)) .
\end{gathered}
$$

Of this sample, 2323 sources had FWHM measurements for both $\mathrm{H} \beta$ and MgII. The lower left panel of Fig. 10 displays the virial FWHM measurements from $\mathrm{H} \beta$ and MgII. The resulting best-fit 

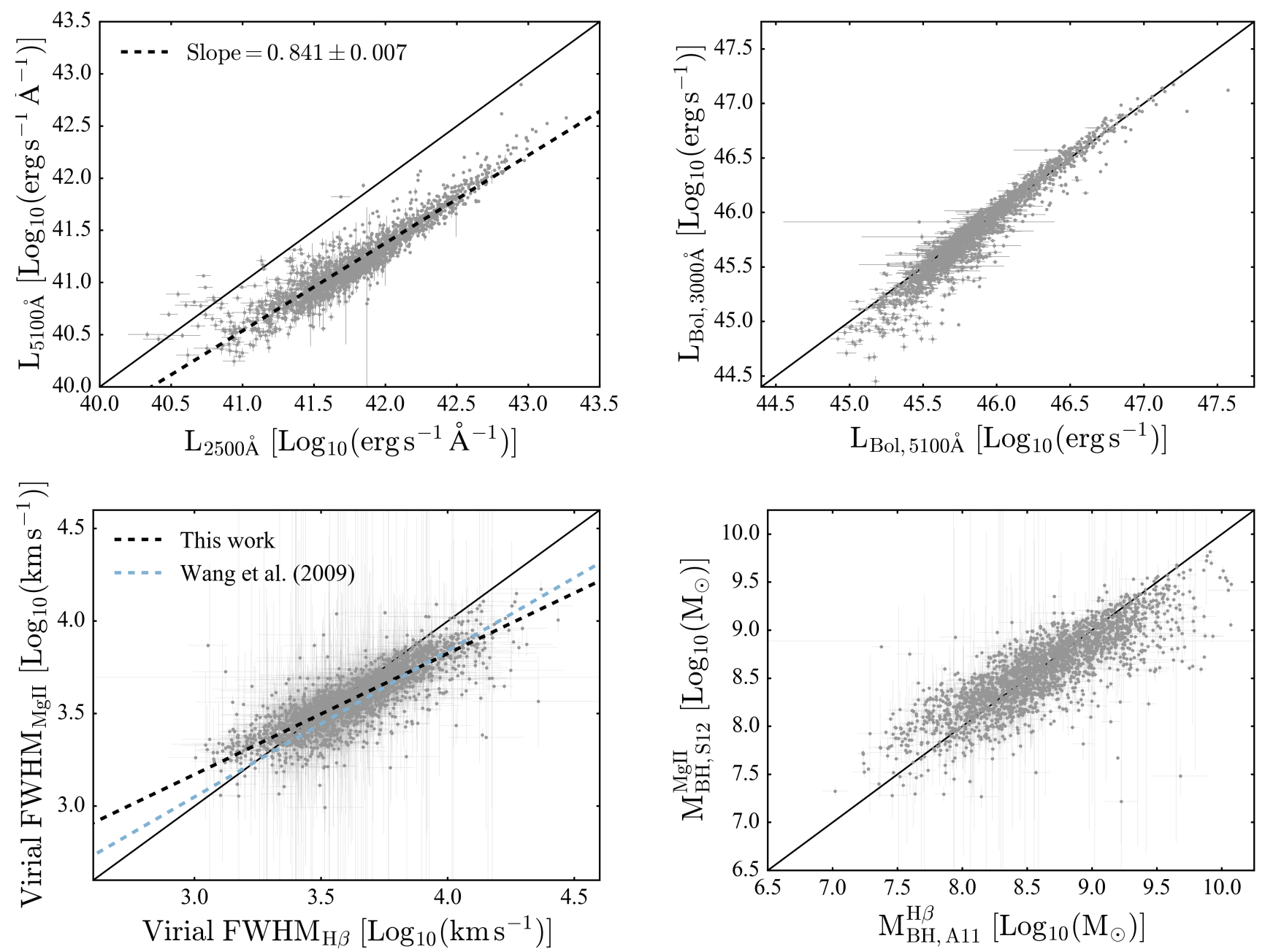

Fig. 10. Comparison between the results obtained from fitting the $\mathrm{H} \beta$ and MgII regions of the spectrum. Upper left: $5100 \AA$ and $2500 \AA$ monochromatic luminosities. Upper right: bolometric luminosities estimated from the $5100 \AA$ and $3000 \AA$ monochromatic luminosities. Lower left: virial FWHM measured using $\mathrm{H} \beta$ and MgII. Lower right: $\mathrm{BH}$ mass estimates derived from $\mathrm{H} \beta$ (using the Assef et al. 2011 calibration) and MgII (using the Shen \& Liu 2012 calibration). In each panel, the solid black line is the unity line.

relation, fit using LINMIX, is

$\log _{10}\left(F W H M_{\mathrm{MgII}}\right)=(0.65 \pm 0.01) \log _{10}\left(F W H M_{\mathrm{H} \beta}\right)+(1.21 \pm 0.04)$

with a regression intrinsic scatter of 0.005 . This deviation from the one-to-one relation has also been observed by Wang et al. (2009), who reported a slope of $0.81 \pm 0.02$, and Shen \& Liu (2012), who found a slope of $0.57 \pm 0.09$. The single-epoch $\mathrm{BH}$ mass relations (Eq. (2)) account for the $F W H M_{\mathrm{MgII}}-$ $F W H M_{\mathrm{H} \beta}$ slope; when the correct $\mathrm{BH}$ mass calibration is used, the $\mathrm{H} \beta$ and $\mathrm{MgII}$ virial FWHM measurements yield $\mathrm{BH}$ masses that are in close agreement (see the lower right panel of Fig. 10).

\section{Sample properties}

Figure 11 presents the comparison between this sample and the full sample of SDSS DR7 AGN with optical spectral properties measured by Shen et al. (2011) in the bolometric luminosity-redshift and bolometric luminosity-BH mass planes. As discussed in Sect. 1, $\mathrm{H}_{\beta}$-derived $\mathrm{BH}$ masses are used where available (shown in blue), while MgII-derived masses are used for the remaining higher-redshift sources (shown in green). The left panel of Fig. 11 shows that this sample populates the lowredshift, high-luminosity region of the parameter space, which is partially due to the high flux threshold of the X-ray selection. From the right panel of Fig. 11 it can be seen that the sample presented in this work appears to be well bounded by the Eddington limit at least up to $M_{\mathrm{BH}} \simeq 10^{9.5} M_{\odot}$.

\subsection{H $\beta$ line components}

Section 3.2 described how the $\mathrm{H} \beta$ line profile was fit with either one, two, three, or four Gaussian components. Figure 12 displays the resulting distribution of $\mathrm{H} \beta$ FWHM measurements (the panels are split based on the number of Gaussian components required to fit the line). There is a clear peak in the distribution at low FWHM associated with the narrow $\mathrm{H} \beta$ core typically measured at a few hundred $\mathrm{km} \mathrm{s}^{-1}$. Above $\sim 1000 \mathrm{~km} \mathrm{~s}^{-1}$ the distribution is bimodal (in the two lower panels) with a large number of sources showing evidence for the "very broad component" (VBC) of $\mathrm{H} \beta$ at $F W H M \geq 10000 \mathrm{~km} \mathrm{~s}^{-1}$ also discussed in Marziani et al. (2010).

It has been suggested that the VBC is emitted from a distinct physical region, and is possibly the result of line emission from the accretion disk (e.g. Bon et al. 2009). If the VBC represents emission from the accretion disk, then a strong VBC may result in a bias towards a higher $\mathrm{BH}$ mass estimate, since the single-epoch method assumes a calibration that is based on 

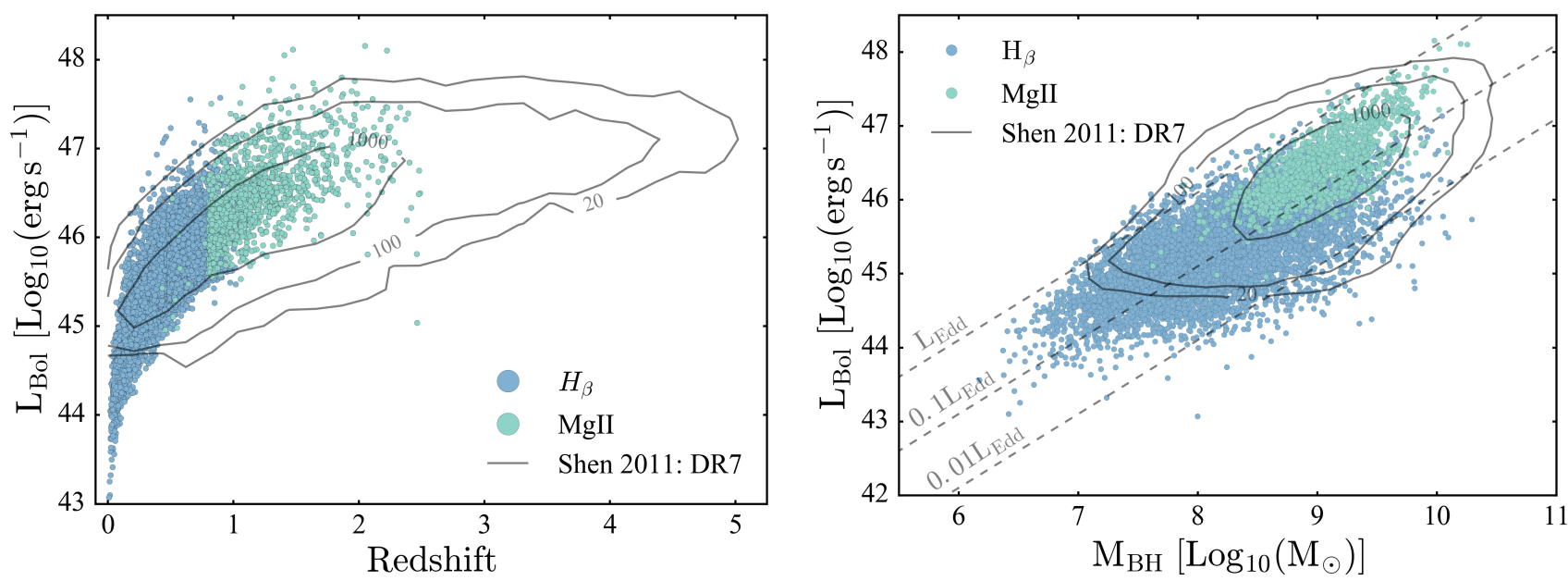

Fig. 11. Bolometric luminosity versus redshift (left panel) and bolometric luminosity versus BH mass (right panel) for the sample presented in this work and the Shen et al. (2011) sample. Sources with $\mathrm{H}_{\beta}$-derived $\mathrm{BH}$ masses are shown in blue, and sources with $\mathrm{MgII}$-derived $\mathrm{BH}$ masses are displayed in green.

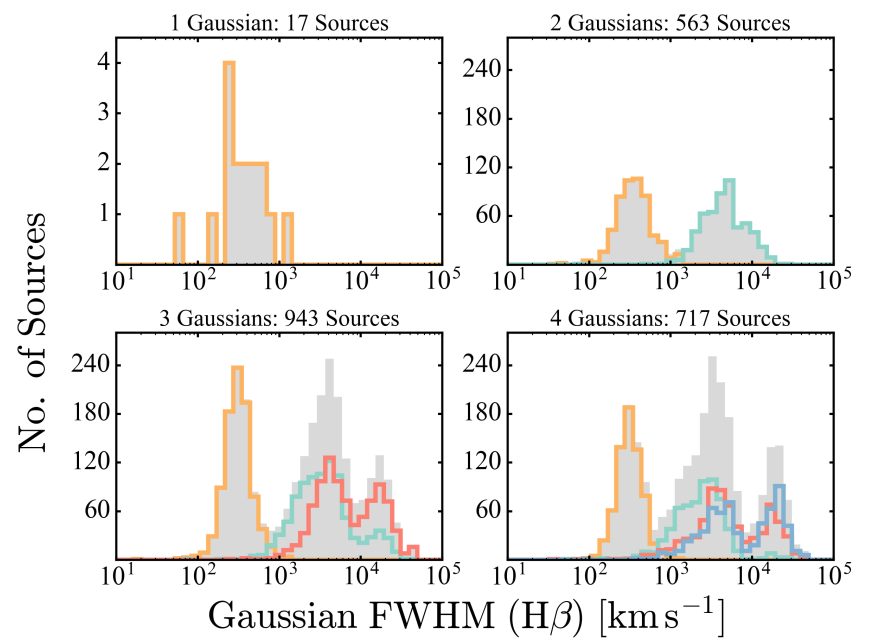

Fig. 12. Distribution of $\mathrm{H} \beta$ Gaussian FWHM values. The panels are split based on the number of Gaussians required to fit the line. The coloured histograms each represent one of up to four possible Gaussians used to fit the $\mathrm{H} \beta$ line. The grey histograms represent the sum of the individual coloured histograms.

the luminosity-BLR radius relation. However, since the kinematics and physical origin of the VBC remains uncertain, detected VBCs have not been excluded from the broad line profiles used to measure the virial FWHM in this analysis (as discussed in Sect. 3.3).

\subsection{This sample in the $4 D$ eigenvector 1 context}

The 4D Eigenvector 1 (4DE1) system (Boroson \& Green 1992; Sulentic et al. 2000, 2011) aims to define a set of parameters that uniquely account for AGN diversity. Two main 4DE1 parameters are the FWHM of the broad component of $\mathrm{H} \beta\left(F W H M \mathrm{H}_{\beta}^{\mathrm{BC}}\right)$ and the strength of the FeII emission relative to that of $\mathrm{H} \beta$, defined as

$R_{\mathrm{FeII}}=F_{\mathrm{FeII}} / F_{\mathrm{H} \beta}$

where $F_{\mathrm{FeII}}$ and $F_{\mathrm{H} \beta}$ are the fluxes of the FeII emission in the 4434-4684 $\AA$ range and broad $\mathrm{H} \beta$ line, respectively. A sample of 2098 sources with measurements of these parameters and reli- able spectral fits $\left(0 \leq \chi_{\nu, \mathrm{H} \beta}^{2} \leq 1.2\right)$ was selected. The left panel of Fig. 13 shows the distribution of this sample in the 4DE1 parameter space (grey). It is expected that a reliable measurement of the FeII component will be difficult for many of the lower $\mathrm{S} / \mathrm{N}$ sources (see Marziani et al. 2003). For this reason, the subset of sources in Fig. 13 with a median $\mathrm{S} / \mathrm{N}$ greater than or equal to 20 per resolution element is also shown (blue). The right panel of Fig. 13 presents the higher $\mathrm{S} / \mathrm{N}$ sources, colour-coded as a function of Eddington ratio. The expected trend of increasing Eddington ratio towards smaller $F W H M \mathrm{H}_{\beta}^{\mathrm{BC}}$ and larger $R_{\mathrm{FeII}}$ is observed for this sample of high-S/N sources. Typically, sources with both high $R_{\mathrm{FeII}}$ and high $F W H M \mathrm{H}_{\beta}^{\mathrm{BC}}$ are not observed. If these sources exist, they may be difficult to detect since strong FeII emission might conceal a faint $\mathrm{H}_{\beta}$ broad component. The potential bias in the 4DE1 plane source distribution due to model limitations and spectral $\mathrm{S} / \mathrm{N}$ is discussed in Sects. 9.1.2 and 9.1.3.

The grey dashed line in the right panel of Fig. 13 indicates the division between population $\mathrm{A}\left(F W H M \mathrm{H}_{\beta}^{\mathrm{BC}} \leq 4000 \mathrm{~km} \mathrm{~s}^{-1}\right)$ and population $\mathrm{B}\left(F W H M \mathrm{H}_{\beta}^{\mathrm{BC}} \geq 4000 \mathrm{~km} \mathrm{~s}^{-1}\right)$ sources in the 4DE1 context (see Sulentic et al. 2011). Population A sources often possess Lorentzian broad line profiles, and it has been suggested that Gaussian fits to population A broad lines will result in an underestimation of the BH mass (see Sulentic et al. 2014).

\subsection{Relationship between AGN X-ray and optical emission}

Quasars exhibit a non-linear relationship between their X-ray and UV emission, usually represented by the $\alpha_{\text {OX }}$ parameter

$\alpha_{\mathrm{OX}}=\frac{\log \left(L_{2 \mathrm{keV}} / L_{2500 \AA}\right)}{\log \left(v_{2 \mathrm{keV}} / v_{2500 \AA}\right)}$

where $L_{2 \mathrm{keV}}, L_{2500 \AA}, v_{2 \mathrm{keV}}$, and $v_{2500 A A}$ are the monochromatic luminosities and frequencies at $2 \mathrm{keV}$ and $2500 \AA$, respectively (Vignali et al. 2003; Strateva et al. 2005; Steffen et al. 2006; Just et al. 2007; Kelly et al. 2008; Green et al. 2009; Young et al. 2009; Lusso et al. 2010). The $\alpha_{\text {OX }}$ parameter is considered to be a proxy for the relative contribution of the UV accretion disk emission and the X-ray emission from the surrounding corona to the total luminosity. In order to study this relationship, a sample of sources with measurements of the $2 \mathrm{keV}$, 

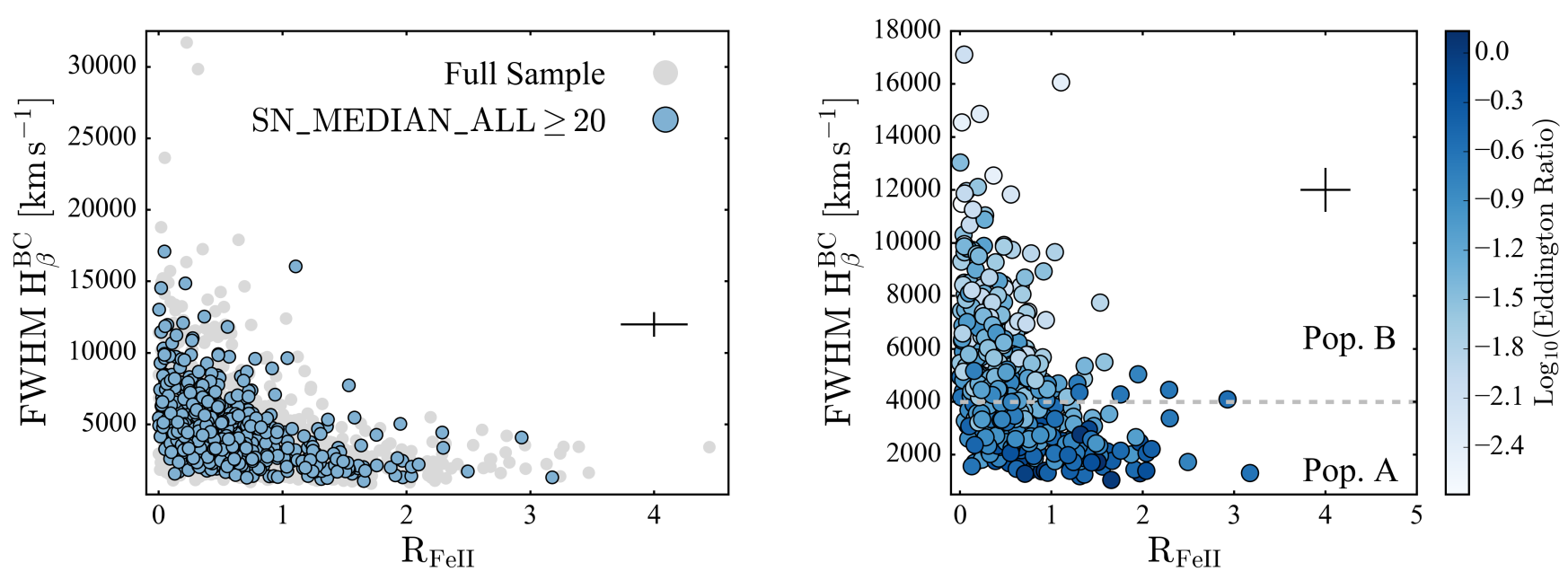

Fig. 13. FWHM of the broad component of $\mathrm{H} \beta$ versus $R_{\mathrm{FeII}}$. The left panel displays the sample described in Sect. 8.2 (grey), and sources with a median spectral $S / N \geq 20$ (blue). The right panel presents the same subsample of high $\mathrm{S} / \mathrm{N}$ sources, colour coded to indicate the trend in Eddington ratio across the distribution. The grey dashed line marks the division between population $\mathrm{A}\left(F W H M \mathrm{H}_{\beta}^{\mathrm{BC}} \leq 4000 \mathrm{~km} \mathrm{~s}^{-1}\right)$ and population $\mathrm{B}$ $\left(F W H M \mathrm{H}_{\beta}^{\mathrm{BC}} \geq 4000 \mathrm{~km} \mathrm{~s}^{-1}\right)$ sources. The size of the typical uncertainties, multiplied by a factor of five, in both variables for the high-S/N subsample is also shown.

$2500 \AA$, and $5100 \AA$ luminosities was selected. For lower redshift sources without spectral coverage of $2500 \AA$, Eq. (4) was used to estimate the $2500 \AA$ luminosity from the $5100 \AA$ luminosity. Extended sources were removed in order to prevent additional scatter in the relationship due to the contribution of the host galaxy. This was done by requiring that the SDSS $g$ band "stellarity"7 (defined as $\mathrm{S}(\mathrm{g})=$ cModelMag_g - psfMag_g) lies between \pm 0.1 . This sample does not contain X-ray sources with more than one potential AllWISE counterpart and therefore avoids cases where the X-ray detection includes emission from more than one object. This selection process resulted in a sample of 4777 sources. Figure 14 shows the $\alpha_{\text {OX }}$ parameter versus the monochromatic luminosity at $2500 \AA$. The $\alpha_{\mathrm{OX}}-L_{2500 \AA}$ relation was fit using LINMIX, which gave the following best-fit result

$\alpha_{\mathrm{OX}}=2.39 \pm 0.16-(0.124 \pm 0.005) \log \left(L_{2500 \AA}\right)$

with a regression intrinsic scatter of 0.0034 . This slope is consistent with previous results from the literature (e.g. Kelly et al. 2008).

\section{Interpreting the data and limitations}

In this section, the reliability and limitations of the sample will be discussed.

\subsection{Measuring the Fell emission}

Distinguishing the FeII component from the continuum emission becomes more difficult when using low $\mathrm{S} / \mathrm{N}$ spectra. In addition, for a given $\mathrm{S} / \mathrm{N}$, it may also be more difficult to detect FeII emission if the intrinsic broadening of the FeII lines is large, since broader, blended FeII emission lines are more likely to be fit by the model as continuum emission (see Marziani et al. 2003). Using simulated AGN spectra, Marziani et al. (2003) estimate the minimum detectable optical FeII emission as a function of $\mathrm{H} \beta$ width for different bins of $\mathrm{S} / \mathrm{N}$.

\footnotetext{
7 For a description of how cModelMag_g and psfMag_g are measured see https://www . sdss .org/dr12/algorithms/magnitudes/.
}

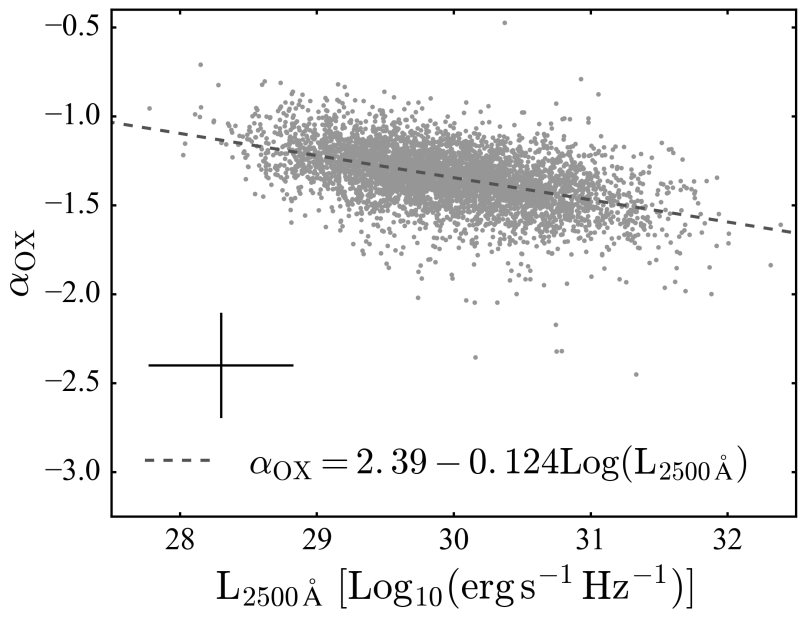

Fig. 14. $\alpha_{\mathrm{OX}}$ versus UV luminosity. The dashed line is the best linear fit to the distribution. The size of the typical uncertainties in both variables is also shown.

A poor fit to the FeII emission may affect the accuracy of the BH mass estimates, since FeII emission can influence measurements of both the broad line width (see Sect. 9.1.1) and the continuum luminosity. FeII emission may also conceal a broad $\mathrm{H} \beta$ component thus biasing a source's position in the 4DE1 plane (Fig. 13). These potential issues are tested in the following three sections.

\subsubsection{Accuracy of the broad emission line FWHM measurements for sources with Fell continuum emission}

A poor fit to the FeII emission may affect the measurement of the broad emission line width. To quantify the magnitude of this effect, the $\mathrm{H} \beta$ fitting script (using four Gaussians to fit $\mathrm{H} \beta$ ) was run with and without the FeII template on a sample of $\sim 400$ randomly selected sources. The fit without the FeII template represents the most extreme case where the FeII emission is completely ignored by the model. Therefore, the change in line 


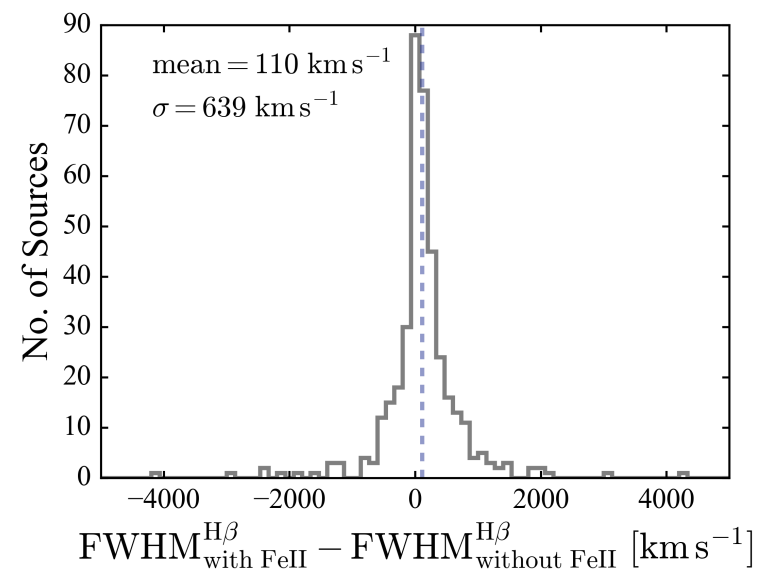

Fig. 15. Comparison of the $\mathrm{H} \beta$ FWHM measurements derived using a model with and without an FeII template. The vertical dashed line shows the mean value of the distribution.

widths measured by these two models should be the upper limit on what can be expected for cases where the FeII fit is inadequate. Figure 15 shows that the line width dispersion induced by ignoring the presence of FeII emission is $\simeq 640 \mathrm{~km} \mathrm{~s}^{-1}$.

\subsubsection{Model limitations in detecting sources in the 4DE1 plane}

Sources with both large $R_{\mathrm{FeII}}$ and large $F W H M \mathrm{H}_{\beta}^{\mathrm{BC}}$ are typically not observed, however, this absence may be due to model limitations; at high $R_{\text {FeII }}$ the broad $\mathrm{H} \beta$ component may be concealed beneath the FeII emission, and therefore may not be detected. The experiment outlined in this section was carried out in order to determine whether the spectral fitting code used in this work would return accurate measurements for sources with high $R_{\mathrm{FeII}}$ and $F W H M \mathrm{H}_{\beta}^{\mathrm{BC}}$ values.

A parameter space defined by $0.1 \leq R_{\mathrm{FeII}}<5$ and $1000 \mathrm{~km} \mathrm{~s}^{-1} \leq F W H M \mathrm{H}_{\beta}^{\mathrm{BC}}<15000 \mathrm{~km} \mathrm{~s}^{-1}$ was divided into a $12 \times 12$ grid. $10 \mathrm{~S} / \mathrm{N}$ bins between 5 and 50 (a representative range for the samples presented in this work) were selected for each point on the grid, and 10 spectra were simulated for each $R_{\mathrm{FeII}}-F W H M \mathrm{H}_{\beta}^{\mathrm{BC}}-S / N$ combination, resulting in 14400 simulated spectra. For the parameters that were fixed in this experiment, the interquartile mean of the best-fit values for the type 1 AGN in this sample were used. The $\mathrm{H} \beta$ line profile was modelled with one narrow and one broad Gaussian. The wavelength range was set to 4420-5500 $\AA$ (as in Sect. 3.2) and the logarithmic wavelength spacing ${ }^{8}$ was set to be equal to that of SDSS spectra;

$\log _{10} \lambda_{i+1}-\log _{10} \lambda_{i}=0.0001$.

These spectra were fit using a version of the $\mathrm{H} \beta$ fitting script which used one narrow and one broad Gaussian component to fit $\mathrm{H} \beta$. The minimum $\mathrm{S} / \mathrm{N}$ required for the fitting script to return the correct $R_{\mathrm{FeII}}$ and $F W H M \mathrm{H}_{\beta}^{\mathrm{BC}}$ combinations was then determined. In order to consider an $R_{\mathrm{FeII}}$ and $F W H M \mathrm{H}_{\beta}^{\mathrm{BC}}$ combination detectable at a given $\mathrm{S} / \mathrm{N}$, at least $7 / 10$ spectra were required to have best fit $R_{\mathrm{FeII}}$ and $F W H M \mathrm{H}_{\beta}^{\mathrm{BC}}$ values that agreed with the input values.

Figure 16 shows the detectable $R_{\mathrm{FeII}}$ and $F W H M \mathrm{H}_{\beta}^{\mathrm{BC}}$ combinations for each point on the grid, along with the minimum

\footnotetext{
8 https://www.sdss.org/dr12/spectro/spectro_basics/
}

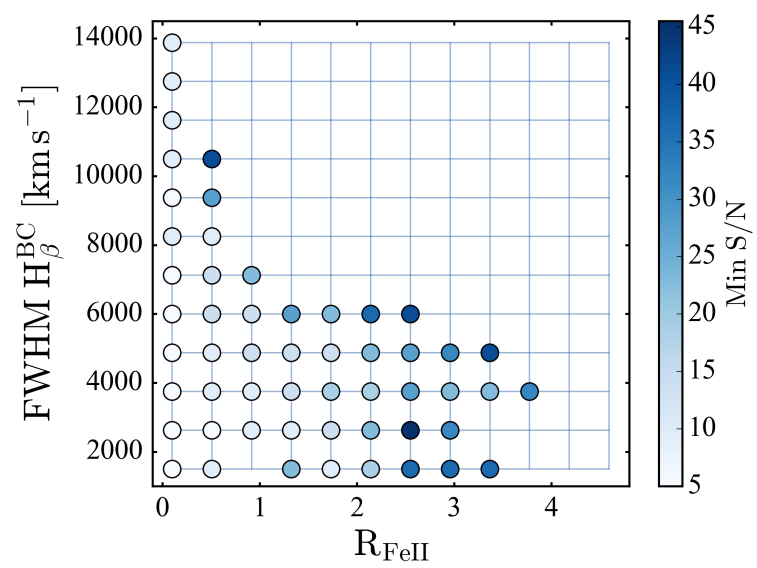

Fig. 16. Minimum $\mathrm{S} / \mathrm{N}$ required to detect a range of $R_{\mathrm{FeII}}$ and $F W H M \mathrm{H}_{\beta}^{\mathrm{BC}}$ combinations. The blue grid indicates the range of the parameter space covered in the simulation described in Sect. 9.1.2. Points on the grid that do not have a minimum $\mathrm{S} / \mathrm{N}$ indicator represent $R_{\text {FeII }}$ and $F W H M \mathrm{H}_{\beta}^{\mathrm{BC}}$ combinations that are not detectable even at the highest $\mathrm{S} / \mathrm{N}$ used in this experiment. Sources detected at these $R_{\mathrm{FeII}}$ and $F W H M \mathrm{H}_{\beta}^{\mathrm{BC}}$ combinations are likely to be spurious (see Fig. 17).

$\mathrm{S} / \mathrm{N}$ required to detect that combination. It is clear from Fig. 16 that at the $\mathrm{S} / \mathrm{N}$ levels available in this sample, a large region of the 4DE1 parameter space would not be detected.

\subsubsection{Bias in the $R_{\text {Fell }}$ and $F W H M H_{\beta}^{B C}$ distribution due to model limitations}

Figure 17 shows the comparison between the simulated and measured $R_{\mathrm{FeII}}$ and $F W H M \mathrm{H}_{\beta}^{\mathrm{BC}}$ values for the highest and lowest $\mathrm{S} / \mathrm{N}$ bins used in Sect. 9.1.2. The left panel of Fig. 17 shows that at low $\mathrm{S} / \mathrm{N}$ the results are clearly biased against high $R_{\mathrm{FeII}}$ and $F W H M \mathrm{H}_{\beta}^{\mathrm{BC}}$ values. At higher $\mathrm{S} / \mathrm{N}$ (Fig. 17, right panel), the accuracy of the lower left quadrant measurements is significantly improved. However, even at $S / N=45.5$ (which is approximately the upper end of the $\mathrm{S} / \mathrm{N}$ distribution of the samples presented in this work) the high $R_{\mathrm{FeII}}-F W H M \mathrm{H}_{\beta}^{\mathrm{BC}}$ measurements deviate significantly from the corresponding "true" values. This may suggest that the L-shaped distribution of sources in the 4DE1 plane (e.g. Fig. 13) is at least in part due to model limitations.

\subsection{Reliability of the single-epoch method for mass estimation}

Assuming that AGN broad emission lines are produced by gas whose motion is dominated by the gravitational potential of the central SMBH, the single-epoch method is expected to produce reliable mass estimates when compared to RM (see Vestergaard \& Peterson 2006), with a systematic uncertainty of 0.3-0.4 dex. However, it is not clear how to measure the virial FWHM of lines that deviate from this norm.

The spectrum shown in the left panel of Fig. 18 is an example of a source which exhibits a double-peaked $\mathrm{H} \beta$ line profile, where a clear inflection point is visible between two velocityshifted broad line components. Double-peaked broad line profiles in $\mathrm{AGN}$ are expected to be the result of emission from the accretion disk (Perez et al. 1988; Chen et al. 1989; Eracleous \& Halpern 1994, 2003; Strateva et al. 2003). Zhang et al. (2007) have found that single-epoch $\mathrm{BH}$ mass estimates obtained from doublepeaked line profiles are significantly larger than $\mathrm{BH}$ mass 

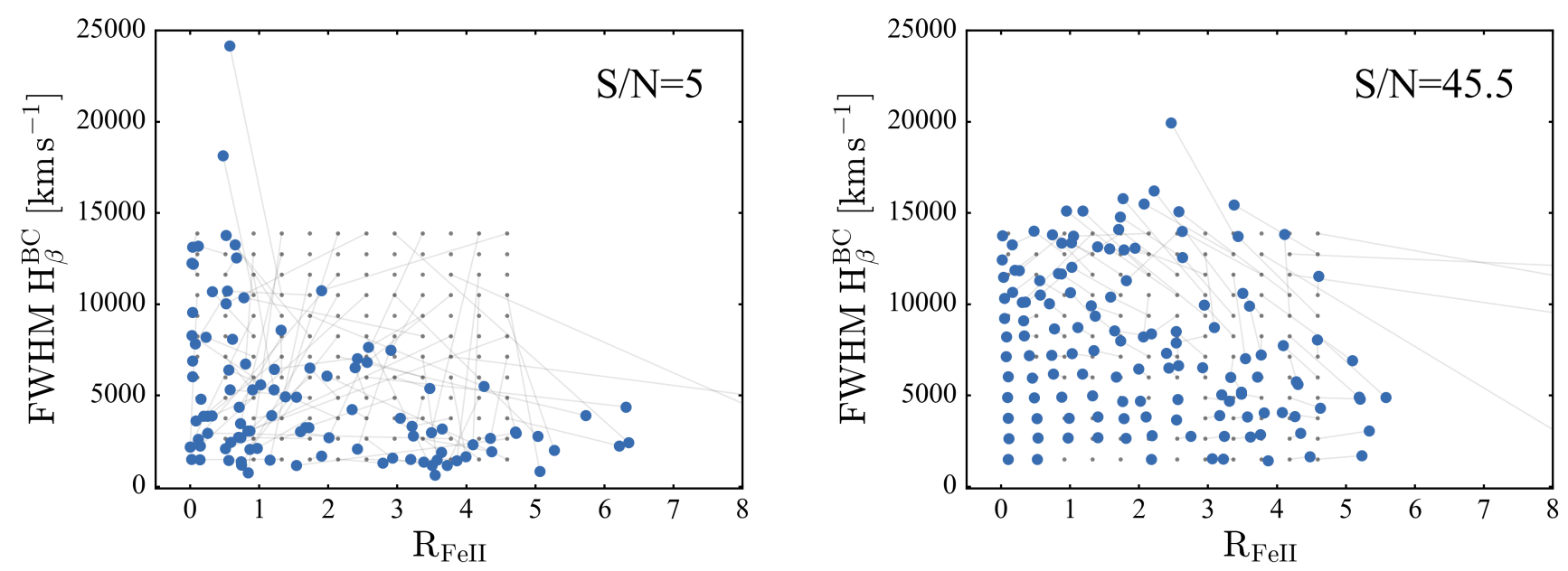

Fig. 17. Comparison between the measured and simulated $R_{\mathrm{FeII}}$ and $F W H M \mathrm{H}_{\beta}^{\mathrm{BC}}$ values for the highest and lowest $\mathrm{S} / \mathrm{N}$ bins used in Sect. 9.1.2. For clarity, each figure displays only one of the ten sets of spectra for that $\mathrm{S} / \mathrm{N}$. The values used to simulate the spectra (grey points) are connected to the corresponding best fit measurements (except for cases where either the broad $\mathrm{H} \beta$ or FeII components were not detected). For clarity, the figures do not show a small number of unphysically high $R_{\text {FeII }}$ measurements.
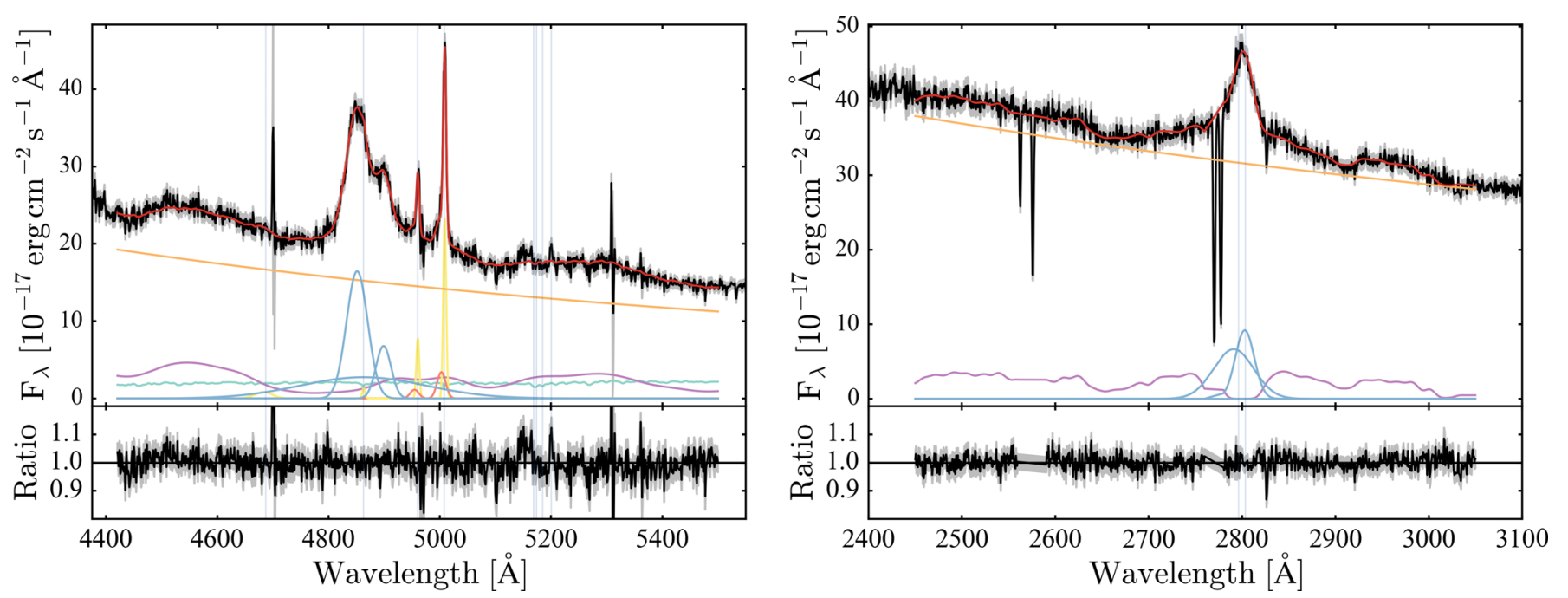

Fig. 18. Left panel: example of a source with a double-peaked $\mathrm{H} \beta$ line profile (plate $=7512$, MJD $=56777$, fiber $=321$ ). Right panel: example of a source showing narrow UV absorption features which have been masked when fitting the model $($ plate $=8188$, MJD $=57348$, fiber $=946)$. See Sect. 9.2 for details.

estimates derived from stellar velocity dispersion measurements. Zhang et al. (2007) suggest that this discrepancy is the result of an overestimation of the BLR radius by the single-epoch mass calibrations for these objects. Therefore, the $\mathrm{BH}$ mass estimates provided in this work for sources which exhibit double-peaked broad emission lines should be treated with caution.

The right panel of Fig. 18 shows an example of narrow absorption in the UV portion of the spectrum caused by intervening absorbing material along the line of sight to the AGN. Sources identified during the visual inspection as having narrow absorption lines have been flagged in the catalogue (column 189; flag_abs). These sources were fit using the model described in Sect. 3.4 with the absorption line regions masked. However, in many cases, the absorption features distort the broad MgII line, and therefore the resulting $\mathrm{BH}$ mass estimates may not be reliable.

\section{Conclusions}

This work presents a catalogue of spectral properties for all SPIDERS type 1 AGN up to SDSS DR14. Visual inspection results were used to select a reliable subsample for spectral analysis, and the spectral regions around $\mathrm{H} \beta$ and $\mathrm{MgII}$ were fit with a multicomponent model. Using the single-epoch method, $\mathrm{BH}$ masses, bolometric luminosities, Eddington ratios, along with additional spectral parameters were measured. A catalogue containing these results has been made available as part of a set of SDSS DR14 value added catalogues. This catalogue also includes the results of a visual inspection of the sample?

Acknowledgements. DC has participated in the International Max Planck Research School on Astrophysics at the Ludwig Maximilians University Munich. DC also acknowledges financial support from the Max Planck Society. DC would also like to thank Riccardo Arcodia and Julien Wolf for many helpful discussions. The authors would like to thank Josephine Reisinger for her contribution to the visual inspection of sources in this sample. Finally, the authors would like to thank the anonymous referee for providing a thorough critique of the paper which greatly improved its content. Funding for the Sloan Digital Sky Survey IV has been provided by the Alfred P. Sloan Foundation, the U.S. Department of Energy Office of Science, and the Participating Institutions. SDSS-IV

\footnotetext{
9 http://www .mpe.mpg.de/XraySurveys/SPIDERS/SPIDERS_
} AGN/ 
acknowledges support and resources from the Center for High-Performance Computing at the University of Utah. The SDSS web site is www.sdss.org. SDSS-IV is managed by the Astrophysical Research Consortium for the Participating Institutions of the SDSS Collaboration including the Brazilian Participation Group, the Carnegie Institution for Science, Carnegie Mellon University, the Chilean Participation Group, the French Participation Group, HarvardSmithsonian Center for Astrophysics, Instituto de Astrofísica de Canarias, The Johns Hopkins University, Kavli Institute for the Physics and Mathematics of the Universe (IPMU)/University of Tokyo, Lawrence Berkeley National Laboratory, Leibniz Institut für Astrophysik Potsdam (AIP), Max-Planck-Institut für Astronomie (MPIA Heidelberg), Max-Planck-Institut für Astrophysik (MPA Garching), Max-Planck-Institut für Extraterrestrische Physik (MPE), National Astronomical Observatories of China, New Mexico State University, New York University, University of Notre Dame, Observatário Nacional/MCTI, The Ohio State University, Pennsylvania State University, Shanghai Astronomical Observatory, United Kingdom Participation Group, Universidad Nacional Autónoma de México, University of Arizona, University of Colorado Boulder, University of Oxford, University of Portsmouth, University of Utah, University of Virginia, University of Washington, University of Wisconsin, Vanderbilt University, and Yale University. Funding for SDSS-III has been provided by the Alfred P. Sloan Foundation, the Participating Institutions, the National Science Foundation, and the U.S. Department of Energy Office of Science. The SDSS-III web site is http://www.sdss3.org/. SDSS-III is managed by the Astrophysical Research Consortium for the Participating Institutions of the SDSS-III Collaboration including the University of Arizona, the Brazilian Participation Group, Brookhaven National Laboratory, Carnegie Mellon University, University of Florida, the French Participation Group, the German Participation Group, Harvard University, the Instituto de Astrofísica de Canarias, the Michigan State/Notre Dame/JINA Participation Group, Johns Hopkins University, Lawrence Berkeley National Laboratory, Max Planck Institute for Astrophysics, Max Planck Institute for Extraterrestrial Physics, New Mexico State University, New York University, Ohio State University, Pennsylvania State University, University of Portsmouth, Princeton University, the Spanish Participation Group, University of Tokyo, University of Utah, Vanderbilt University, University of Virginia, University of Washington, and Yale University. Funding for the SDSS and SDSS-II has been provided by the Alfred P. Sloan Foundation, the Participating Institutions, the National Science Foundation, the U.S. Department of Energy, the National Aeronautics and Space Administration, the Japanese Monbukagakusho, the Max Planck Society, and the Higher Education Funding Council for England. The SDSS Web Site is http://www.sdss.org/. The SDSS is managed by the Astrophysical Research Consortium for the Participating Institutions. The Participating Institutions are the American Museum of Natural History, Astrophysical Institute Potsdam, University of Basel, University of Cambridge, Case Western Reserve University, University of Chicago, Drexel University, Fermilab, the Institute for Advanced Study, the Japan Participation Group, Johns Hopkins University, the Joint Institute for Nuclear Astrophysics, the Kavli Institute for Particle Astrophysics and Cosmology, the Korean Scientist Group, the Chinese Academy of Sciences (LAMOST), Los Alamos National Laboratory, the Max-Planck-Institute for Astronomy (MPIA), the MaxPlanck-Institute for Astrophysics (MPA), New Mexico State University, Ohio State University, University of Pittsburgh, University of Portsmouth, Princeton University, the United States Naval Observatory, and the University of Washington. This publication makes use of data products from the Wide-field Infrared Survey Explorer, which is a joint project of the University of California, Los Angeles, and the Jet Propulsion Laboratory/California Institute of Technology, and NEOWISE, which is a project of the Jet Propulsion Laboratory/California Institute of Technology. WISE and NEOWISE are funded by the National Aeronautics and Space Administration. Plot colours were in part based on www. ColorBrewer.org, by Cynthia A. Brewer, Penn State. The TOPCAT tool (Taylor 2005) was used during this work.

\section{References}

Abolfathi, B., Aguado, D. S., Aguilar, G., et al. 2018, ApJS, 235, 42 Albareti, F. D., Allende Prieto, C., Almeida, A., et al. 2017, ApJS, 233, 25 Anderson, S. F., Margon, B., Voges, W., et al. 2007, AJ, 133, 313

Arnaud, K. A. 1996, in Astronomical Data Analysis Software and Systems V, eds. G. H. Jacoby, \& J. Barnes, ASP Conf. Ser., 101, 17

Assef, R. J., Denney, K. D., Kochanek, C. S., et al. 2011, ApJ, 742, 93

Bahcall, J. N., Kozlovsky, B.-Z., \& Salpeter, E. E. 1972, ApJ, 171, 467

Baskin, A., \& Laor, A. 2005, MNRAS, 356, 1029

Bentz, M. C., \& Katz, S. 2015, PASP, 127, 67

Bentz, M. C., Peterson, B. M., Pogge, R. W., Vestergaard, M., \& Onken, C. A. 2006, ApJ, 644, 133

Bentz, M. C., Peterson, B. M., Netzer, H., Pogge, R. W., \& Vestergaard, M. 2009a, ApJ, 697, 160

Bentz, M. C., Walsh, J. L., Barth, A. J., et al. 2009b, ApJ, 705, 199
Blandford, R. D., \& McKee, C. F. 1982, ApJ, 255, 419

Blanton, M. R., Bershady, M. A., Abolfathi, B., et al. 2017, AJ, 154, 28

Boller, T., Freyberg, M. J., Trümper, J., et al. 2016, A\&A, 588, A103

Bon, E., Popović, L. Č., Gavrilović, N., La Mura, G., \& Mediavilla, E. 2009, MNRAS, 400, 924

Boroson, T. 2005, AJ, 130, 381

Boroson, T. A., \& Green, R. F. 1992, ApJS, 80, 109

Brunner, H., Cappelluti, N., Hasinger, G., et al. 2008, A\&A, 479, 283

Calderone, G., Nicastro, L., Ghisellini, G., et al. 2017, MNRAS, 472, 4051

Capriotti, E. R., Foltz, C. B., \& Peterson, B. M. 1982, ApJ, 261, 35

Cardelli, J. A., Clayton, G. C., \& Mathis, J. S. 1989, ApJ, 345, 245

Chen, K., Halpern, J. P., \& Filippenko, A. V. 1989, ApJ, 339, 742

Civano, F., Marchesi, S., Comastri, A., et al. 2016, ApJ, 819, 62

Coatman, L., Hewett, P. C., Banerji, M., et al. 2017, MNRAS, 465, 2120

Cutri, R. M., et al. 2013, VizieR Online Data Catalog: II/328

Dawson, K. S., Schlegel, D. J., Ahn, C. P., et al. 2013, AJ, 145, 10

Dawson, K. S., Kneib, J.-P., Percival, W. J., et al. 2016, AJ, 151, 44

Denney, K. D. 2012, ApJ, 759, 44

Denney, K. D., Peterson, B. M., Dietrich, M., Vestergaard, M., \& Bentz, M. C. 2009, ApJ, 692, 246

Dwelly, T., Salvato, M., Merloni, A., et al. 2017, MNRAS, 469, 1065

Eisenstein, D. J., Weinberg, D. H., Agol, E., et al. 2011, AJ, 142, 72

Eracleous, M., \& Halpern, J. P. 1994, ApJS, 90, 1

Eracleous, M., \& Halpern, J. P. 2003, ApJ, 599, 886

Fotopoulou, S., Salvato, M., Hasinger, G., et al. 2012, ApJS, 198, 1

Gaskell, C. M., \& Sparke, L. S. 1986, ApJ, 305, 175

Gebhardt, K., Bender, R., Bower, G., et al. 2000, ApJ, 539, L13

Georgakakis, A., \& Nandra, K. 2011, MNRAS, 414, 992

Georgakakis, A., Nandra, K., Laird, E. S., Aird, J., \& Trichas, M. 2008, MNRAS, 388, 1205

Giavalisco, M., Ferguson, H. C., Koekemoer, A. M., et al. 2004, ApJ, 600, L93

Green, P. J., Aldcroft, T. L., Richards, G. T., et al. 2009, ApJ, 690, 644

Grogin, N. A., Conselice, C. J., Chatzichristou, E., et al. 2005, ApJ, 627, L97

Gunn, J. E., Siegmund, W. A., Mannery, E. J., et al. 2006, AJ, 131, 2332

Ilbert, O., Arnouts, S., Le Floc'h, E., et al. 2015, A\&A, 579, A2

Just, D. W., Brandt, W. N., Shemmer, O., et al. 2007, ApJ, 665, 1004

Kaspi, S., Smith, P. S., Netzer, H., et al. 2000, ApJ, 533, 631

Kaspi, S., Maoz, D., Netzer, H., et al. 2005, ApJ, 629, 61

Kelly, B. C. 2007, ApJ, 665, 1489

Kelly, B. C., Bechtold, J., Trump, J. R., Vestergaard, M., \& Siemiginowska, A. 2008, ApJS, 176, 355

Kormendy, J., \& Richstone, D. 1995, ARA\&A, 33, 581

Kraft, R. P., Burrows, D. N., \& Nousek, J. A. 1991, ApJ, 374, 344

Laird, E. S., Nandra, K., Georgakakis, A., et al. 2009, ApJS, 180, 102

LaMassa, S. M., Urry, C. M., Cappelluti, N., et al. 2016, ApJ, 817, 172

Liu, Z., Merloni, A., Georgakakis, A., et al. 2016, MNRAS, 459, 1602

Luo, B., Brandt, W. N., Xue, Y. Q., et al. 2017, ApJS, 228, 2

Lusso, E., Comastri, A., Vignali, C., et al. 2010, A\&A, 512, A34

Magorrian, J., Tremaine, S., Richstone, D., et al. 1998, AJ, 115, 2285

Marchesi, S., Civano, F., Elvis, M., et al. 2016a, ApJ, 817, 34

Marchesi, S., Lanzuisi, G., Civano, F., et al. 2016b, ApJ, 830, 100

Markwardt, C. B. 2009, in Astronomical Data Analysis Software and Systems XVIII, eds. D. A. Bohlender, D. Durand, \& P. Dowler, ASP Conf. Ser., 411, 251

Marziani, P., Sulentic, J. W., Zamanov, R., et al. 2003, ApJS, 145, 199

Marziani, P., Sulentic, J. W., Negrete, C. A., et al. 2010, MNRAS, 409, 1033

McLure, R. J., \& Jarvis, M. J. 2002, MNRAS, 337, 109

Mejía-Restrepo, J. E., Trakhtenbrot, B., Lira, P., \& Netzer, H. 2018, MNRAS, 478, 1929

Menzel, M.-L., Merloni, A., Georgakakis, A., et al. 2016, MNRAS, 457, 110

Merloni, A., Predehl, P., \& Becker, W. 2012, ArXiv e-prints [arXiv:1209. 3114]

Merritt, D., \& Ferrarese, L. 2001, ApJ, 547, 140

Nandra, K., Laird, E. S., Aird, J. A., et al. 2015, ApJS, 220, 10

Pâris, I., Petitjean, P., Ross, N. P., et al. 2017, A\&A, 597, A79

Park, D., Woo, J.-H., Denney, K. D., \& Shin, J. 2013, ApJ, 770, 87

Pereira-Santaella, M., Alonso-Herrero, A., Santos-Lleo, M., et al. 2011, A\&A, 535, A93

Perez, E., Penston, M. V., Tadhunter, C., Mediavilla, E., \& Moles, M. 1988, MNRAS, 230, 353

Persic, M., \& Rephaeli, Y. 2002, A\&A, 382, 843

Peterson, B. M. 1993, PASP, 105, 247

Peterson, B. M., \& Wandel, A. 2000, ApJ, 540, L13

Peterson, B. M., Ferrarese, L., Gilbert, K. M., et al. 2004, ApJ, 613, 682

Pierce, C. M., Lotz, J. M., Laird, E. S., et al. 2007, ApJ, 660, L19

Plotkin, R. M., Anderson, S. F., Brandt, W. N., et al. 2010, AJ, 139, 390

Polletta, M., Tajer, M., Maraschi, L., et al. 2007, ApJ, 663, 81

Predehl, P., Andritschke, R., \& Babyshkin, V. 2016, in Space Telescopes and Instrumentation 2016: Ultraviolet to Gamma Ray, Proc. SPIE, 9905, 99051K 
Ranalli, P., Comastri, A., \& Setti, G. 2003, A\&A, 399, 39

Richards, G. T., Vanden Berk, D. E., Reichard, T. A., et al. 2002, AJ, 124,

Richards, G. T., Lacy, M., Storrie-Lombardi, L. J., et al. 2006, ApJS, 166, 470

Runnoe, J. C., Brotherton, M. S., Shang, Z., \& DiPompeo, M. A. 2013, MNRAS 434, 848

Salvato, M., Buchner, J., Budavári, T., et al. 2018, MNRAS, 473, 4937

Saxton, R. D., Read, A. M., Esquej, P., et al. 2008, A\&A, 480, 611

Schlegel, D. J., Finkbeiner, D. P., \& Davis, M. 1998, ApJ, 500, 525

Schneider, D. P., Richards, G. T., Hall, P. B., et al. 2010, AJ, 139, 2360

Schwarz, G. 1978, Ann. Stat., 6, 461

Scoville, N., Aussel, H., Brusa, M., et al. 2007, ApJS, 172, 1

Shen, Y. 2013, Bull. Astron. Soc. India, 41, 61

Shen, Y., \& Liu, X. 2012, ApJ, 753, 125

Shen, Y., Richards, G. T., Strauss, M. A., et al. 2011, ApJS, 194, 45

Shen, Y., Brandt, W. N., Dawson, K. S., et al. 2015, ApJS, 216, 4

Silva, L., Granato, G. L., Bressan, A., \& Danese, L. 1998, ApJ, 509, 103

Smee, S. A., Gunn, J. E., Uomoto, A., et al. 2013, AJ, 146, 32

Steffen, A. T., Strateva, I., Brandt, W. N., et al. 2006, AJ, 131, 2826

Storey, P. J., \& Zeippen, C. J. 2000, MNRAS, 312, 813

Strateva, I. V., Strauss, M. A., Hao, L., et al. 2003, AJ, 126, 1720
Strateva, I. V., Brandt, W. N., Schneider, D. P., Vanden Berk, D. G., \& Vignali, C. 2005, AJ, 130, 387

Sulentic, J. W., Zwitter, T., Marziani, P., \& Dultzin-Hacyan, D. 2000, ApJ, 536, L5

Sulentic, J., Marziani, P., \& Zamfir, S. 2011, Baltic Astron., 20, 427

Sulentic, J. W., Marziani, P., Olmo, A. D., \& Plauchu-Frayn, I. 2014, Adv. Space Res., 54, 1406

Taylor, M. B. 2005, in Astronomical Data Analysis Software and Systems XIV, eds. P. Shopbell, M. Britton, \& R. Ebert, ASP Conf. Ser., 347, 29

Trakhtenbrot, B., \& Netzer, H. 2012, MNRAS, 427, 3081

Tremaine, S., Gebhardt, K., Bender, R., et al. 2002, ApJ, 574, 740

Vestergaard, M. 2002, ApJ, 571, 733

Vestergaard, M., \& Peterson, B. M. 2006, ApJ, 641, 689

Vestergaard, M., \& Wilkes, B. J. 2001, ApJS, 134, 1

Vignali, C., Brandt, W. N., Schneider, D. P., et al. 2003, AJ, 125, 2876

Voges, W., Aschenbach, B., Boller, T., et al. 1999, A\&A, 349, 389

Wang, J.-G., Dong, X.-B., Wang, T.-G., et al. 2009, ApJ, 707, 1334

Wright, E. L., Eisenhardt, P. R. M., Mainzer, A. K., et al. 2010, AJ, 140, 1868

York, D. G., Adelman, J., Anderson, Jr., J. E., et al. 2000, AJ, 120, 1579

Young, M., Elvis, M., \& Risaliti, G. 2009, ApJS, 183, 17

Zhang, X.-G., Dultzin-Hacyan, D., \& Wang, T.-G. 2007, MNRAS, 376, 1335 


\section{Appendix A: Catalogue column description}

The results from the spectral fitting code have been compiled into a single dataset published as an SDSS DR14 value added catalogue. A description of the columns included in the catalogue is given below. Catalogue entries that are either undetermined or not relevant for a given source are set to "-99". Columns that provide information on the X-ray detections have been taken from Saxton et al. (2008) and Boller et al. (2016).

1. xray_detection: Flag indicating whether the X-ray source was detected in the 2RXS or XMMSL1 survey.

2. name: IAU name of the X-ray detection.

3. RA: Right ascension of the X-ray detection (J2000) [degrees].

4. Dec: Declination of the X-ray detection (J2000) [degrees].

5. ExiML: Existence likelihood of the 2RXS detection.

6. ExpTime_2RXS: Exposure time (2RXS) [s].

7. DET_ML: Detection likelihood of the XMMSL1 detection in the $0.2-12 \mathrm{keV}$ range.

8. ExpTime_XMMSL: Exposure time (XMMSL1) [s].

9. f_2RXS: Flux in the $0.1-2.4 \mathrm{keV}$ range (2RXS) $\left[\mathrm{erg} \mathrm{cm} \mathrm{cm}^{-2} \mathrm{~s}^{-1}\right]$.

10. errf_2RXS: Uncertainty in the flux in the $0.1-2.4 \mathrm{keV}$ range (2RXS) [erg cm $\mathrm{cm}^{-2} \mathrm{~s}^{-1}$.

11. fden_2RXS: Flux density at $2 \mathrm{keV}$ [erg cm $\left.\mathrm{cm}^{-2} \mathrm{~s}^{-1} \mathrm{~Hz}^{-1}\right]$.

12. errfden_2RXS: Uncertainty in the flux density at $2 \mathrm{keV}$ (2RXS) [ $\left.\mathrm{erg} \mathrm{cm}^{-2} \mathrm{~s}^{-1} \mathrm{~Hz}^{-1}\right]$.

13. 1_2RXS: Luminosity in the $0.1-2.4 \mathrm{keV}$ range (2RXS) $\left[\operatorname{erg~s}^{-1}\right]$.

14. errl_2RXS: Uncertainty in the luminosity in the 0.1$2.4 \mathrm{keV}$ range (2RXS) [erg s$\left.{ }^{-1}\right]$.

15. $12 \mathrm{keV} \_2 \mathrm{RXS}$ : Monochromatic luminosity at $2 \mathrm{keV}(2 \mathrm{RXS})$ $\left[\mathrm{erg} \mathrm{s}^{-1} \mathrm{~Hz}^{-1}\right.$ ].

16. errl2keV_2RXS: Uncertainty in the monochromatic luminosity at $2 \mathrm{keV}$ (2RXS) [ $\mathrm{erg} \mathrm{s}^{-1} \mathrm{~Hz}^{-1}$ ].

17. f_XMMSL: Flux in the $0.2-12 \mathrm{keV}$ range (XMMSL1; from Saxton et al. 2008) $\left[\mathrm{erg} \mathrm{cm}^{-2} \mathrm{~s}^{-1}\right]$.

18. errf_XMMSL: Uncertainty in the flux in the $0.2-12 \mathrm{keV}$ range (XMMSL1; from Saxton et al. 2008) $\left[\mathrm{erg} \mathrm{cm}^{-2} \mathrm{~s}^{-1}\right.$ ].

19. 1_XMMSL: Luminosity in the $0.2-12 \mathrm{keV}$ range (XMMSL1; from Saxton et al. 2008) [ $\mathrm{erg} \mathrm{s}^{-1}$ ].

20. errl_XMMSL: Uncertainty in the luminosity in the 0.2-12 keV range (XMMSL1; from Saxton et al. 2008) $\left[\mathrm{erg} \mathrm{s}^{-1}\right]$.

21. Plate: SDSS plate number.

22. MJD: MJD that the SDSS spectrum was taken.

23. FiberID: SDSS fiber identification.

24. DR14_RUN2D: Spectroscopic reprocessing number.

25. DR14_PLUGRA: Right ascension of the drilled fiber position [degrees].

26. DR14_PLUGDEC: Declination of the drilled fiber position [degrees].

27. redshift: Source redshift based on the visual inspection results.

28. CLASS_BEST: Source classification based on the visual inspection results.

29. CONF_BEST: Visual inspection redshift and classification confidence flag.

30. DR14_ZWARNING: Warning flag for SDSS spectra.

31. DR14_SNMEDIANALL: Median S/N per pixel of the spectrum.

32. Instrument: Flag indicating which spectrograph was used (SDSS or BOSS) to measure the spectrum.
33. norm1_mgII: Normalisation of the first Gaussian used to fit the MgII line $\left[10^{-17} \mathrm{erg} \mathrm{cm}^{-2} \mathrm{~s}^{-1} \AA^{-1}\right]$.

34. errnorm1_mgII: Uncertainty in the normalisation of the first Gaussian used to fit the MgII line $\left[10^{-17} \mathrm{erg} \mathrm{cm}^{-2} \mathrm{~s}^{-1} \AA^{-1}\right]$.

35. peak1_mgII: Wavelength of the peak of the first Gaussian used to fit the MgII line [A] .

36. errpeak1_mgII: Uncertainty in the wavelength of the peak of the first Gaussian used to fit the MgII line $[\AA]$.

37. width1_mgII: Width of the first Gaussian used to fit the MgII line $[\AA]$.

38. errwidth1_mgII: Uncertainty in the width of the first Gaussian used to fit the MgII line [Å].

39. fwhm1_mgII: FWHM of the first Gaussian used to fit the MgII line $\left[\mathrm{km} \mathrm{s}^{-1}\right]$.

40. errfwhm1_mgII: Uncertainty in the FWHM of the first Gaussian used to fit the MgII line $\left[\mathrm{km} \mathrm{s}^{-1}\right]$.

41. shift1_mgII: Wavelength shift of the peak of the first Gaussian used to fit the MgII line relative to the rest-frame wavelength $[\AA]$.

42. norm2_mgII: Normalisation of the second Gaussian used to fit the MgII line $\left[10^{-17} \mathrm{erg} \mathrm{cm}^{-2} \mathrm{~s}^{-1} \AA^{-1}\right]$.

43. errnorm2_mgII: Uncertainty in the normalisation of the second Gaussian used to fit the MgII line $\left[10^{-17} \mathrm{erg} \mathrm{cm}^{-2} \mathrm{~s}^{-1} \AA^{-1}\right]$.

44. peak2_mgII: Wavelength of the peak of the second Gaussian used to fit the MgII line [Å].

45. errpeak2_mgII: Uncertainty in the wavelength of the peak of the second Gaussian used to fit the MgII line $[\AA]$.

46. width2_mgII: Width of the second Gaussian used to fit the MgII line [̊̊].

47. errwidth2_mgII: Uncertainty in the width of the second

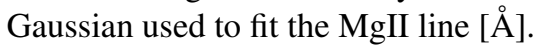

48. fwhm2_mgII: FWHM of the second Gaussian used to fit the $\mathrm{MgII}$ line $\left[\mathrm{km} \mathrm{s}^{-1}\right]$.

49. errfwhm2_mgII: Uncertainty in the FWHM of the second Gaussian used to fit the MgII line [ $\left.\mathrm{km} \mathrm{s}^{-1}\right]$.

50. shift2_mgII: Wavelength shift of the peak of the second Gaussian used to fit the MgII line relative to the rest-frame wavelength [̊].

51. norm3_mgII: Normalisation of the third Gaussian used to fit the MgII line $\left[10^{-17} \mathrm{erg} \mathrm{cm}^{-2} \mathrm{~s}^{-1} \AA^{-1}\right]$.

52. errnorm3_mgII: Uncertainty in the normalisation of the third Gaussian used to fit the MgII line $\left[10^{-17} \mathrm{erg} \mathrm{cm}^{-2} \mathrm{~s}^{-1} \AA^{-1}\right]$.

53. peak3_mgII: Wavelength of the peak of the third Gaussian used to fit the MgII line [̊̊].

54. errpeak3_mgII: Uncertainty in the wavelength of the peak of the third Gaussian used to fit the MgII line [ $\mathrm{A}]$.

55. width3_mgII: Width of the third Gaussian used to fit the MgII line [Å].

56. errwidth3_mgII: Uncertainty in the width of the third Gaussian used to fit the MgII line [Å].

57. fwhm3_mgII: FWHM of the third Gaussian used to fit the MgII line $\left[\mathrm{km} \mathrm{s}^{-1}\right]$.

58. errfwhm3_mgII: Uncertainty in the FWHM of the third Gaussian used to fit the MgII line $\left[\mathrm{km} \mathrm{s}^{-1}\right]$.

59. shift3_mgII: Wavelength shift of the peak of the third Gaussian used to fit the MgII line relative to the rest-frame wavelength $[\AA]$. 
60. norm_heII: Normalisation of the Gaussian used to fit the HeII line $\left[10^{-17} \mathrm{erg} \mathrm{cm}^{-2} \mathrm{~s}^{-1} \AA^{-1}\right]$.

61. errnorm_heII: Uncertainty in the normalisation of the Gaussian used to fit the HeII line $\left[10^{-17} \mathrm{erg} \mathrm{cm}^{-2} \mathrm{~s}^{-1} \AA^{-1}\right]$.

62. peak_heII: Wavelength of the peak of the Gaussian used to fit the HeII line $[\AA]$.

63. errpeak_heII: Uncertainty in the wavelength of the peak of the Gaussian used to fit the HeII line [Å].

64. width_heII: Width of the Gaussian used to fit the HeII line [A] .

65. errwidth_heII: Uncertainty in the width of the Gaussian used to fit the HeII line $[\AA]$.

66. fwhm_heII: FWHM of the Gaussian used to fit the HeII line $\left[\mathrm{km} \mathrm{s}^{-1}\right]$.

67. errfwhm_heII: Uncertainty in the FWHM of the Gaussian used to fit the HeII line $\left[\mathrm{km} \mathrm{s}^{-1}\right]$.

68. shift_heII: Wavelength shift of the peak of the Gaussian used to fit the HeII line relative to the rest-frame wavelength $[\AA]$.

69. norm1_hb: Normalisation of the first Gaussian used to fit the $\mathrm{H} \beta$ line $\left[10^{-17} \mathrm{erg} \mathrm{cm}^{-2} \mathrm{~s}^{-1} \AA^{-1}\right]$.

70. errnorm1_hb: Uncertainty in the normalisation of the first Gaussian used to fit the $\mathrm{H} \beta$ line $\left[10^{-17} \mathrm{erg} \mathrm{cm}^{-2} \mathrm{~s}^{-1} \AA^{-1}\right]$.

71. peak1_hb: Wavelength of the peak of the first Gaussian used to fit the $\mathrm{H} \beta$ line $[\AA]$.

72. errpeak1_hb: Uncertainty in the wavelength of the peak of the first Gaussian used to fit the $\mathrm{H} \beta$ line $[\AA]$.

73. width1_hb: Width of the first Gaussian used to fit the $\mathrm{H} \beta$ line $[\AA]$.

74. errwidth1_hb: Uncertainty in the width of the first Gaussian used to fit the $\mathrm{H} \beta$ line $[\AA]$.

75. fwhm1_hb: FWHM of the first Gaussian used to fit the $\mathrm{H} \beta$ line $\left[\mathrm{km} \mathrm{s}^{-1}\right]$.

76. errfwhm1_hb: Uncertainty in the FWHM of the first Gaussian used to fit the $\mathrm{H} \beta$ line $\left[\mathrm{km} \mathrm{s}^{-1}\right]$.

77. shift1_hb: Wavelength shift of the peak of the first Gaussian used to fit the $\mathrm{H} \beta$ line relative to the rest-frame wavelength [A] .

78. norm2_hb: Normalisation of the second Gaussian used to fit the $\mathrm{H} \beta$ line $\left[10^{-17} \mathrm{erg} \mathrm{cm}^{-2} \mathrm{~s}^{-1} \AA^{-1}\right]$.

79. errnorm 2 hb: Uncertainty in the normalisation of the second Gaussian used to fit the $\mathrm{H} \beta$ line $\left[10^{-17} \mathrm{erg} \mathrm{cm}^{-2} \mathrm{~s}^{-1} \AA^{-1}\right]$.

80. peak2_hb: Wavelength of the peak of the second Gaussian used to fit the $\mathrm{H} \beta$ line $[\AA ̊]$.

81. errpeak2_hb: Uncertainty in the wavelength of the peak of the second Gaussian used to fit the $\mathrm{H} \beta$ line $[\AA]$.

82. width2_hb: Width of the second Gaussian used to fit the $\mathrm{H} \beta$ line $[\AA]$.

83. errwidth2_hb: Uncertainty in the width of the second Gaussian used to fit the $\mathrm{H} \beta$ line $[\AA]$.

84. fwhm2_hb: FWHM of the second Gaussian used to fit the $\mathrm{H} \beta$ line $\left[\mathrm{km} \mathrm{s}^{-1}\right]$.

85. errfwhm2_hb: Uncertainty in the FWHM of the second Gaussian used to fit the $\mathrm{H} \beta$ line $\left[\mathrm{km} \mathrm{s}^{-1}\right]$.

86. shift 2 hb: Wavelength shift of the peak of the second Gaussian used to fit the $\mathrm{H} \beta$ line relative to the rest-frame wavelength $[\AA]$.

87. norm3_hb: Normalisation of the third Gaussian used to fit the $\mathrm{H} \beta$ line $\left[10^{-17} \mathrm{erg} \mathrm{cm}^{-2} \mathrm{~s}^{-1} \AA^{-1}\right]$.

88. errnorm3_hb: Uncertainty in the normalisation of the third Gaussian used to fit the $\mathrm{H} \beta$ line $\left[10^{-17} \mathrm{erg} \mathrm{cm}^{-2} \mathrm{~s}^{-1} \AA^{-1}\right]$.
89. peak3_hb: Wavelength of the peak of the third Gaussian used to fit the $\mathrm{H} \beta$ line $[\AA]$.

90. errpeak3_hb: Uncertainty in the wavelength of the peak of the third Gaussian used to fit the $\mathrm{H} \beta$ line $[\AA]$.

91. width3_hb: Width of the third Gaussian used to fit the $\mathrm{H} \beta$ line $[\AA]$.

92. errwidth3_hb: Uncertainty in the width of the third Gaussian used to fit the $\mathrm{H} \beta$ line $[\AA]$.

93. fwhm3_hb: FWHM of the third Gaussian used to fit the $\mathrm{H} \beta$ line $\left[\mathrm{km} \mathrm{s}^{-1}\right]$.

94. errfwhm3_hb: Uncertainty in the FWHM of the third Gaussian used to fit the $\mathrm{H} \beta$ line $\left[\mathrm{km} \mathrm{s}^{-1}\right]$.

95. shift3_hb: Wavelength shift of the peak of the third Gaussian used to fit the $\mathrm{H} \beta$ line relative to the rest-frame wavelength $[\AA]$.

96. norm4_hb: Normalisation of the fourth Gaussian used to fit the $\mathrm{H} \beta$ line $\left[10^{-17} \mathrm{erg} \mathrm{cm}^{-2} \mathrm{~s}^{-1} \AA^{-1}\right]$.

97. errnorm4_hb: Uncertainty in the normalisation of the fourth Gaussian used to fit the $\mathrm{H} \beta$ line $\left[10^{-17} \mathrm{erg} \mathrm{cm}^{-2} \mathrm{~s}^{-1} \AA^{-1}\right]$.

98. peak4_hb: Wavelength of the peak of the fourth Gaussian used to fit the $\mathrm{H} \beta$ line $[\AA]$.

99. errpeak4_hb: Uncertainty in the wavelength of the peak of the fourth Gaussian used to fit the $\mathrm{H} \beta$ line $[\AA]$.

100. width4_hb: Width of the fourth Gaussian used to fit the $\mathrm{H} \beta$ line $[\AA]$.

101. errwidth4_hb: Uncertainty in the width of the fourth Gaussian used to fit the $\mathrm{H} \beta$ line $[\AA]$.

102. fwhm4_hb: FWHM of the fourth Gaussian used to fit the $\mathrm{H} \beta$ line $\left[\mathrm{km} \mathrm{s}^{-1}\right]$.

103. errfwhm4_hb: Uncertainty in the FWHM of the fourth Gaussian used to fit the $\mathrm{H} \beta$ line $\left[\mathrm{km} \mathrm{s}^{-1}\right]$.

104. shift4_hb: Wavelength shift of the peak of the fourth Gaussian used to fit the $\mathrm{H} \beta$ line relative to the rest-frame wavelength $[\AA]$.

105. norm1_OIII4959: Normalisation of first Gaussian used to fit the [OIII]4959 line $\left[10^{-17} \mathrm{erg} \mathrm{cm}^{-2} \mathrm{~s}^{-1} \AA^{-1}\right]$.

106. errnorm1_OIII4959: Uncertainty in the normalisation of first Gaussian used to fit the [OIII]4959 line $\left[10^{-17} \mathrm{erg} \mathrm{cm}^{-2} \mathrm{~s}^{-1} \AA^{-1}\right]$.

107. peak1_OIII4959: Wavelength of the peak of the first Gaussian used to fit the [OIII]4959 line [̊̊].

108. errpeak1_OIII4959: Uncertainty in the wavelength of the peak of the first Gaussian used to fit the [OIII]4959 line [A] .

109. width1_OIII4959: Width of the first Gaussian used to fit the [OIII]4959 line [̊].

110. errwidth1_OIII4959: Uncertainty in the width of the first Gaussian used to fit the [OIII]4959 line [̊̊].

111. fwhm1_OIII4959: FWHM of the first Gaussian used to fit the [OIII]4959 line $\left[\mathrm{km} \mathrm{s}^{-1}\right]$.

112. errfwhm1_OIII4959: Uncertainty in the FWHM of the first Gaussian used to fit the [OIII]4959 line $\left[\mathrm{km} \mathrm{s}^{-1}\right]$.

113. shift1_OIII4959: Wavelength shift of the peak of the first Gaussian used to fit the [OIII]4959 line relative to the restframe wavelength $[\AA]$.

114. norm2_OIII4959: Normalisation of second Gaussian used to fit the [OIII]4959 line $\left[10^{-17} \mathrm{erg} \mathrm{cm}^{-2} \mathrm{~s}^{-1} \AA^{-1}\right]$.

115. errnorm2_OIII4959: Uncertainty in the normalisation of second Gaussian used to fit the [OIII]4959 line $\left[10^{-17} \mathrm{erg} \mathrm{cm}^{-2} \mathrm{~s}^{-1} \AA^{-1}\right]$. 
116. peak2_OIII4959: Wavelength of the peak of the second Gaussian used to fit the [OIII]4959 line [Å].

117. errpeak2_OIII4959: Uncertainty in the wavelength of the peak of the second Gaussian used to fit the [OIII]4959 line [A] .

118. width2_OIII4959: Width of the second Gaussian used to fit the [OIII]4959 line $[\AA]$.

119. errwidth2_OIII4959: Uncertainty in the width of the second Gaussian used to fit the [OIII]4959 line [^].

120. fwhm2_OIII4959: FWHM of the second Gaussian used to fit the [OIII]4959 line $\left[\mathrm{km} \mathrm{s}^{-1}\right]$.

121. errfwhm2_OIII4959: Uncertainty in the FWHM of the second Gaussian used to fit the [OIII]4959 line $\left[\mathrm{km} \mathrm{s}^{-1}\right]$.

122. shift2_OIII4959: Wavelength shift of the peak of the second Gaussian used to fit the [OIII]4959 line relative to the rest-frame wavelength $[\AA]$.

123. norm1_OIII5007: Normalisation of first Gaussian used to fit the [OIII]5007 line $\left[10^{-17} \mathrm{erg} \mathrm{cm}^{-2} \mathrm{~s}^{-1} \AA^{-1}\right]$.

124. errnorm1_OIII5007: Uncertainty in the normalisation of first Gaussian used to fit the [OIII]5007 line $\left[10^{-17} \mathrm{erg} \mathrm{cm}^{-2} \mathrm{~s}^{-1} \AA^{-1}\right]$.

125. peak1_OIII5007: Wavelength of the peak of the first Gaus-

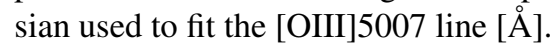

126. errpeak1_OIII5007: Uncertainty in the wavelength of the peak of the first Gaussian used to fit the [OIII]5007 line [A].

127. width1_OIII5007: Width of the first Gaussian used to fit the [OIII] 5007 line $[\AA ̊]$.

128. errwidth1_OIII5007: Uncertainty in the width of the first Gaussian used to fit the [OIII]5007 line [A]].

129. fwhm1_OIII5007: FWHM of the first Gaussian used to fit the [OIII]5007 line $\left[\mathrm{km} \mathrm{s}^{-1}\right]$.

130. errfwhm1_OIII5007: Uncertainty in the FWHM of the first Gaussian used to fit the [OIII]5007 line $\left[\mathrm{km} \mathrm{s}^{-1}\right]$.

131. shift1_OIII5007: Wavelength shift of the peak of the first Gaussian used to fit the [OIII]5007 line relative to the restframe wavelength $[\AA]$.

132. norm2_OIII5007: Normalisation of second Gaussian used to fit the [OIII]5007 line $\left[10^{-17} \mathrm{erg} \mathrm{cm}^{-2} \mathrm{~s}^{-1} \AA^{-1}\right]$.

133. errnorm2_OIII5007: Uncertainty in the normalisation of second Gaussian used to fit the [OIII]5007 line $\left[10^{-17} \mathrm{erg} \mathrm{cm}^{-2} \mathrm{~s}^{-1} \AA^{-1}\right]$.

134. peak2_OIII5007: Wavelength of the peak of the second Gaussian used to fit the [OIII]5007 line [Å].

135. errpeak2_OIII5007: Uncertainty in the wavelength of the peak of the second Gaussian used to fit the [OIII]5007 line [A] .

136. width2_OIII5007: Width of the second Gaussian used to fit the $[\mathrm{OIII}] 5007$ line $[\AA]$.

137. errwidth2_OIII5007: Uncertainty in the width of the second Gaussian used to fit the [OIII]5007 line [^].

138. fwhm2_OIII5007: FWHM of the second Gaussian used to fit the [OIII] 5007 line $\left[\mathrm{km} \mathrm{s}^{-1}\right]$.

139. errfwhm2_OIII5007: Uncertainty in the FWHM of the second Gaussian used to fit the [OIII]5007 line $\left[\mathrm{km} \mathrm{s}^{-1}\right]$.

140. shift2_OIII5007: Wavelength shift of the peak of the second Gaussian used to fit the [OIII]5007 line relative to the rest-frame wavelength $[\AA]$.

141. norm_pl1: Normalisation of the power law fit to the MgII continuum region $\left[10^{-17} \mathrm{erg} \mathrm{cm}^{-2} \mathrm{~s}^{-1} \AA^{-1}\right]$.

142. errnorm_pl1: Uncertainty in the normalisation of the power law fit to the $\mathrm{MgII}$ continuum region $\left[10^{-17} \mathrm{erg} \mathrm{cm}^{-2} \mathrm{~s}^{-1} \AA^{-1}\right]$.
143. slope_pl1: Slope of the power law fit to the MgII continuum region.

144. errslope_pl1: Uncertainty in the slope of the power law fit to the MgII continuum region.

145. norm_pl2: Normalisation of the power law fit to the $\mathrm{H} \beta$ continuum region $\left[10^{-17} \mathrm{erg} \mathrm{cm}^{-2} \mathrm{~s}^{-1} \AA^{-1}\right]$.

146. errnorm_pl2: Uncertainty in the normalisation of the power law fit to the $\mathrm{H} \beta$ continuum region $\left[10^{-17} \mathrm{erg} \mathrm{cm}^{-2} \mathrm{~s}^{-1} \AA^{-1}\right]$.

147. slope_pl2: Slope of the power law fit to the $\mathrm{H} \beta$ continuum region.

148. errslope_pl2: Uncertainty in the slope of the power law fit to the $\mathrm{H} \beta$ continuum region.

149. norm_gal1: Normalisation of the galaxy template used to fit the MgII continuum region $\left[10^{-17} \mathrm{erg} \mathrm{cm}^{-2} \mathrm{~s}^{-1} \AA^{-1}\right]$.

150. errnorm_gal1: Uncertainty in the normalisation of the galaxy template used to fit the MgII continuum region $\left[10^{-17} \mathrm{erg} \mathrm{cm}^{-2} \mathrm{~s}^{-1} \AA^{-1}\right]$.

151. norm_gal2: Normalisation of the galaxy template used to fit the $\mathrm{H} \beta$ continuum region $\left[10^{-17} \mathrm{erg} \mathrm{cm}^{-2} \mathrm{~s}^{-1} \AA^{-1}\right]$.

152. errnorm_gal2: Uncertainty in the normalisation of the galaxy template used to fit the $\mathrm{H} \beta$ continuum region $\left[10^{-17} \mathrm{erg} \mathrm{cm}^{-2} \mathrm{~s}^{-1} \AA^{-1}\right]$.

153. norm_feII1: Normalisation of the iron template used to fit the $\mathrm{MgII}$ continuum region $\left[10^{-17} \mathrm{erg} \mathrm{cm}^{-2} \mathrm{~s}^{-1} \AA^{-1}\right]$.

154. errnorm_feII1: Uncertainty in the normalisation of the iron template used to fit the $\mathrm{MgII}$ continuum region $\left[10^{-17} \mathrm{erg} \mathrm{cm}^{-2} \mathrm{~s}^{-1} \AA^{-1}\right]$.

155. norm_feII2: Normalisation of the iron template used to fit the $\mathrm{H} \beta$ continuum region $\left[10^{-17} \mathrm{erg} \mathrm{cm}^{-2} \mathrm{~s}^{-1} \AA^{-1}\right]$.

156. errnorm_feII2: Uncertainty in the normalisation of the iron template used to fit the $\mathrm{H} \beta$ continuum region $\left[10^{-17} \mathrm{erg} \mathrm{cm}^{-2} \mathrm{~s}^{-1} \AA^{-1}\right]$.

157. fwhm_feII1: FWHM of the Gaussian kernel convolved with the iron template used to fit the MgII continuum region [A].

158. errfwhm_feII1: Uncertainty in the FWHM of the Gaussian kernel convolved with the iron template used to fit the MgII continuum region $[\AA]$.

159. fwhm_feII2: FWHM of the Gaussian kernel convolved with the iron template used to fit the $\mathrm{H} \beta$ continuum region [A].

160. errfwhm_feII2: Uncertainty in the FWHM of the Gaussian kernel convolved with the iron template used to fit the $\mathrm{H} \beta$ continuum region $[\AA]$.

161. r_feII: Flux ratio of the 4434-4684 $\AA$ FeII emission to the broad component of $\mathrm{H} \beta$.

162. OIII_Hbeta_ratio: Flux ratio of [OIII] $5007 \AA$ to $\mathrm{H} \beta$.

163. virialfwhm_mgII: FWHM of the MgII broad line profile $\left[\mathrm{km} \mathrm{s}^{-1}\right]$.

164. errvirialfwhm_mgII: Uncertainty in the FWHM of the MgII broad line profile $\left[\mathrm{km} \mathrm{s}^{-1}\right]$.

165. virialfwhm_hb: FWHM of the $\mathrm{H} \beta$ broad line profile $\left[\mathrm{km} \mathrm{s}^{-1}\right]$.

166. errvirialfwhm_hb: Uncertainty in the FWHM of the $\mathrm{H} \beta$ broad line profile $\left[\mathrm{km} \mathrm{s}^{-1}\right]$.

167. mgII_chi: Reduced chi-squared $\left(\chi_{v}^{2}\right)$ of the fit to the MgII region.

168. hb_chi: Reduced chi-squared $\left(\chi_{v}^{2}\right)$ of the fit to the $\mathrm{H} \beta$ region.

169. 1_2500: Monochromatic luminosity at $2500 \AA$ $\left[\operatorname{erg~s}^{-1} \AA^{-1}\right.$ ]. 
170. errl_2500: Uncertainty in the monochromatic luminosity at $2500 \AA\left[\operatorname{erg~s}^{-1} \AA^{-1}\right]$.

171. 1_3000: Monochromatic luminosity at $3000 \AA$ [erg s ${ }^{-1} \AA^{-1}$ ].

172. errl_3000: Uncertainty in the monochromatic luminosity at

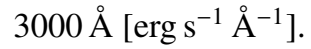

173. 1_5100: Monochromatic luminosity at $5100 \AA$ $\left[\operatorname{erg~s}^{-1} \AA^{-1}\right.$ ].

174. errl_5100: Uncertainty in the monochromatic luminosity at $5100 \AA\left[\mathrm{erg} \mathrm{s}^{-1} \AA^{-1}\right]$.

175. 1_bol1: Bolometric luminosity derived from the monochromatic luminosity at $3000 \AA$ [ $\mathrm{erg} \mathrm{s}^{-1}$ ].

176. errl_bol1: Uncertainty in the bolometric luminosity derived from the monochromatic luminosity at $3000 \AA$ [erg s${ }^{-1}$ ].

177. 1_bol2: Bolometric luminosity derived from the monochromatic luminosity at $5100 \AA$ [erg s${ }^{-1}$ ].

178. errl_bol2: Uncertainty in the bolometric luminosity derived from the monochromatic luminosity at $5100 \AA$ $\left[\mathrm{erg} \mathrm{s}^{-1}\right]$.

179. $\operatorname{logBHMVP} \_$hb: $\mathrm{BH}$ mass derived from the $\mathrm{H} \beta$ line using the Vestergaard \& Peterson (2006) calibration $\left[\log _{10}\left(M_{\odot}\right)\right]$.
180. errlogBHMVP_hb: Uncertainty in the BH mass derived from the $\mathrm{H} \beta$ line using the Vestergaard \& Peterson (2006) calibration $\left[\log _{10}\left(M_{\odot}\right)\right]$.

181. $\log$ BHMA_hb: $\mathrm{BH}$ mass derived from the $\mathrm{H} \beta$ line using the Assef et al. (2011) calibration $\left[\log _{10}\left(M_{\odot}\right)\right]$.

182. errlogBHMA_hb: Uncertainty in the $\mathrm{BH}$ mass derived from the $\mathrm{H} \beta$ line using the Assef et al. (2011) calibration $\left[\log _{10}\left(M_{\odot}\right)\right]$.

183. $\log$ BHMS_mgII: BH mass derived from the MgII line using the Shen \& Liu (2012) calibration $\left[\log _{10}\left(M_{\odot}\right)\right]$.

184. errlogBHMS_mgII: Uncertainty in the BH mass derived from the MgII line using the Shen \& Liu (2012) calibration $\left[\log _{10}\left(M_{\odot}\right)\right]$.

185. 1_edd1: Eddington luminosity based on the $\mathrm{BH}$ mass estimate derived using the Shen \& Liu (2012) calibration $\left[\operatorname{erg~s}^{-1}\right]$.

186. 1_edd2: Eddington luminosity based on the BH mass estimate derived using the Assef et al. (2011) calibration [erg s${ }^{-1}$ ].

187. edd_ratio1: Eddington ratio defined as 1_bol1/1_edd1.

188. edd_ratio2: Eddington ratio defined as 1_bol2/1_edd2.

189. flag_abs: Flag indicating whether or not strong absorption lines have been observed in the spectrum. flag_abs is set to either 0 (no absorption present) or 1 (absorption present). 